University of Nebraska - Lincoln

DigitalCommons@University of Nebraska - Lincoln

\title{
A phase field model of deformation twinning: Nonlinear theory and numerical simulations
}

J. D. Clayton

US Army Research Laboratory, john.d.clayton1@us.army.mil

J. Knap

US Army Research Laboratory

Follow this and additional works at: https://digitalcommons.unl.edu/usarmyresearch

Part of the Operations Research, Systems Engineering and Industrial Engineering Commons

Clayton, J. D. and Knap, J., "A phase field model of deformation twinning: Nonlinear theory and numerical simulations" (2011). US Army Research. 116.

https://digitalcommons.unl.edu/usarmyresearch/116

This Article is brought to you for free and open access by the U.S. Department of Defense at DigitalCommons@University of Nebraska - Lincoln. It has been accepted for inclusion in US Army Research by an authorized administrator of DigitalCommons@University of Nebraska - Lincoln. 


\title{
A phase field model of deformation twinning: Nonlinear theory and numerical simulations
}

\author{
J.D. Clayton ${ }^{\mathrm{a}, *}$, J. Knap ${ }^{\mathrm{b}}$ \\ ${ }^{\text {a } H i g h ~ R a t e ~ M e c h a n i c s ~ a n d ~ F a i l u r e ~ B r a n c h, ~ R D R L-W M P-B, ~ U S ~ A r m y ~ R e s e a r c h ~ L a b o r a t o r y, ~ A b e r d e e n ~ P r o v i n g ~ G r o u n d, ~ M D, ~ 21005-5066, ~ U S A ~}$ \\ ${ }^{\mathrm{b}}$ Computational Science and Engineering Branch, RDRL-CIH-C, US Army Research Laboratory, Aberdeen Proving Ground, MD, 21005-5066, USA
}

\section{A R T I C L E I N F O}

Article history:

Received 2 August 2010

Received in revised form

21 December 2010

Accepted 22 December 2010

Available online 30 December 2010

Communicated by A. Mikhailov

\section{Keywords: \\ Phase field}

Nonlinear elasticity

Energy minimization

Twinning

Crystals

Magnesium

\begin{abstract}
A B S T R A C T
A continuum phase field theory and corresponding numerical solution methods are developed to describe deformation twinning in crystalline solids. An order parameter is associated with the magnitude of twinning shear, i.e., the lattice transformation associated with twinning. The general theory addresses the following physics: large deformations, nonlinear anisotropic elastic behavior, and anisotropic phase boundary energy. The theory is applied towards prediction of equilibrium phenomena in the athermal and non-dissipative limit, whereby equilibrium configurations of an externally stressed crystal are obtained via incremental minimization of a free energy functional. Outcomes of such calculations are elastic fields (e.g., displacement, strain, stress, and strain energy density) and the order parameter field that describes the size and shape of energetically stable twin(s). Numerical simulations of homogeneous twin nucleation in magnesium single crystals demonstrate fair agreement between phase field solutions and available analytical elasticity solutions. Results suggest that critical far-field displacement gradients associated with nucleation of a twin embryo of minimum realistic size are $4.5 \%-5.0 \%$, with particular values of applied shear strain and equilibrium shapes of the twin somewhat sensitive to far-field boundary conditions and anisotropy of twin boundary surface energy.
\end{abstract}

Published by Elsevier B.V.

\section{Introduction}

Phase field models have been successfully applied towards many problems in continuum physics. At each point in the problem domain, one or more phase field variables describe the state of the substance. Conserved phase field variables are typically related to composition, e.g., molar or mass fractions of atoms or molecules. Non-conserved variables include, but are not limited to, order parameters associated with crystal structure, e.g., its symmetry and/or lattice orientation. The present work deals only with non-conserved variables, i.e., order parameters. In this context, the term "phase" denotes a certain crystal structure or lattice configuration. In regions of uniform phase, order parameters take on discrete values of zero or one. In interfacial regions, order parameters enable interpolation between pure phases. In addition to depending on usual mechanical and thermal state variables (e.g., strain and temperature), the local free energy density of a substance generally depends on local value(s) of order parameter(s) and spatial gradients of order parameter(s). Such a prescription enables representation of surface energies of phase boundaries associated with order parameter gradients in interfacial regions.

\footnotetext{
* Corresponding author.

E-mail address: john.d.clayton1@us.army.mil (J.D. Clayton).
}

Pioneering treatments of thermodynamics and kinetics of heterogeneous material systems in the context of phase field models were forwarded, respectively, by Cahn and Hilliard [1] and Allen and Cahn [2]. A fundamental ansatz [2] often prescribed in traditional phase field modeling is that a material system will tend to evolve towards a state of minimum free energy, subject to boundary constraints imposed on the system. Concepts for modeling multiphase systems were advanced by Steinbach et al. [3] and Steinbach and Apel [4]. Fried and Gurtin [5] and Gurtin [6] developed order parameter theories incorporating configurational forces or micro-force balances in the context of geometrically nonlinear continuum mechanics and thermodynamics. Review articles are available that describe various numerical techniques and applications $[7,8]$.

In the present work, phase field theory is used to describe deformation twinning, also known as mechanical twinning, defined as twinning induced by mechanical stresses [9-11]. Hereafter deformation twinning is simply referred to as "twinning". The transformation strain associated with twinning is a simple shear. Across the habit plane, the orientation of the Bravais lattice differs by a reflection or rotation depending on the kind of twin under consideration. The sheared and re-oriented crystal is termed the "twin", while the region of crystal that maintains its original orientation is termed the "parent" or the "matrix". Twinning often takes place by coordinated movement of partial dislocations (i.e., twinning dislocations) and/or shuffles of some but not all atoms comprising the 
twin. A finite energy can be associated with the twin boundary, typically estimated on the order of the appropriate stacking fault energy [12]. More generally, the interfacial energy of a growing or shrinking twin also includes elastic and core energies of twinning dislocations comprising such interfaces $[9,13,14]$.

Continuum mechanics models for deformation twinning have been developed in the context of crystal plasticity theory $[15,16]$, wherein at each continuum material point, the volume/mass fraction of a particular twin system is evolved via a kinetic relation usually involving a resolved shear stress criterion. Intended goals of such models include predictions of macroscopic stress-strain behavior and crystallographic texture. These models are generally unable to predict detailed twin morphologies at the nanometer scale. Sharp interface models have been developed to address kinetics of twin growth $[17,18]$. Such models are capable of predicting twin morphology, but application of these theories requires prescription of kinetic laws for motion of twin boundary interfaces, and their numerical solutions require advanced computational methods such as level sets [18] for resolution of surfaces of discontinuity.

Continuum phase field approaches have been suggested elsewhere for twin growth kinetics $[19,20]$ invoking the TimeDependent Ginzburg-Landau (TDGL) approach for energy minimization. A different approach is pursued in the theory and numerical solution techniques developed in the present paper. The general theory developed here addresses the following physics: potential activity of one or more twin systems, large deformations, nonlinear elastic behavior, and elastic and surface anisotropy. Order parameter(s) are related to magnitude(s) of twinning shear (i.e., transformation strain and rotation) at a material point. Investigated in this work are equilibrium phenomena in the null temperature (i.e., $0 \mathrm{~K}$ ) and quasi-static limits that can be addressed via energy minimization and thermodynamic stability principles. Dissipation and time scales associated with growth kinetics are not formally addressed, nor are acoustic waves. The present approach is somewhat analogous to lattice statics treatments of dislocation mechanics [21,22], wherein dislocation slip in the $0 \mathrm{~K}$ limit is addressed without consideration of atomic vibrations associated with dissipated heat from defect motion. Quasi-static approaches have similarly been used to study twinning in the context of empirical pair potentials [23], empirical many-body potentials [24], and density functional theory (DFT) [25]. Idesman et al. [26] developed a quasi-static continuum approach for modeling elastic-plastic materials undergoing martensitic phase transitions and twinning. Koslowski et al. [27] formulated a phase field theory of dislocation mechanics incorporating a non-convex Peierls potential and energy minimization concepts. The advantage of the present approach over TDGL methods is that material parameters associated with time scales for interfacial motion do not enter the model and need not be measured experimentally. This is an important consideration for modeling of deformation twinning since the propagation speed of twin boundaries can be difficult to measure, and could even be supersonic if the driving stress is sufficiently large [17,28], though twin propagation speeds in the subsonic regime have also been observed [29]. Modeling of twin growth kinetics at realistic time scales in the former case would seem to require resolution of stress dynamics, i.e., wave propagation and possible shock wave phenomena.

The present application of the theory considers homogeneous twin nucleation in an otherwise defect-free single crystal subjected to far-field stress [30-33]. For given far-field boundary conditions, the minimum stable size and equilibrium shape of a twin embryo are dictated by competition between elastic strain energy and surface energy associated with the twin-parent boundary. The theory is implemented in a finite element code that seeks minima of a free energy functional that depends on deformation gradient and order parameter fields. When the surface energy is idealized as isotropic, the only material parameters required are the characteristic twinning shear and geometry (known from the crystal structure), the elastic constants, the twin boundary energy, and a characteristic length associated with the equilibrium twin boundary thickness. Numerical simulations are used to investigate criteria for twin nucleation and growth depending on critical resolved shear stress, twin boundary energy, and far-field boundary constraints. Specifically considered in this paper are $\langle 10 \overline{1} 1\rangle\{\overline{1} 012\}$ twins in magnesium $(\mathrm{Mg})$ single crystals $[13,14,34,23]$.

The remainder of this paper proceeds as follows. Section 2 presents the geometrically nonlinear phase field theory for mechanical twinning. Section 3 presents a geometric linearization of the theory, wherein deformations are assumed small as in conventional linear elasticity. The model described in Section 3 is still nonlinear in the sense that the total free energy of the material is addressed by a potential non-quadratic in the order parameter. Section 4 presents numerical methods used to obtain solutions of boundary value problems, specifically finite element methods with free energy minimization proceeding via a conjugate gradient algorithm. Section 5 presents model predictions for twin nucleation in $\mathrm{Mg}$ single crystals. Conclusions follow in Section 6.

Notation of continuum mechanics is used $[5,6,10]$. Real numbers are $\mathbb{R}$. Vectors and higher-order tensors are written in bold font; scalars and components of vectors and tensors are written in italic font. When indicial notation is used, summation proceeds over repeated indices. To simplify notation, vectors and tensors are referred to a fixed Cartesian frame of reference, with indices in the subscript position. The scalar product of vectors $\mathbf{a}$ and $\mathbf{b}$ is $\mathbf{a} \cdot \mathbf{b}=a_{A} b_{A}=a_{1} b_{1}+a_{2} b_{2}+a_{3} b_{3}$ in a threedimensional vector space. The outer product is $(\mathbf{a} \otimes \mathbf{b})_{A B}=a_{A} b_{B}$. Juxtaposition implies summation over one set of adjacent indices, e.g., $(\mathbf{A B})_{A B}=A_{A C} B_{C B}$. The colon denotes summation over two sets of indices; e.g., $\mathbf{A}: \mathbf{B}=A_{A B} B_{A B}$ and $(\mathbf{C}: \mathbf{E})_{A B}=C_{A B C D} E_{C D}$. The transpose of a matrix is indicated by a $T$ superscript, e.g., $A_{A B}^{T}=A_{B A}$. The inverse operation is denoted by a -1 superscript, and the inverse-transpose operation is denoted by a $-T$ superscript, e.g., $\mathbf{A}^{-T}=\left(\mathbf{A}^{T}\right)^{-1}=\left(\mathbf{A}^{-1}\right)^{T}$.

\section{Geometrically nonlinear theory}

In what follows in Section 2, a phase field theory is developed for an elastic body with a single twin system and describable by a single order parameter. Extension of the theory to elastic bodies with multiple twin systems and multiple order parameters is considered in Appendix B.

\subsection{Order parameter}

Let $\Omega \subset \mathbb{R}^{3}$ be a reference configuration of a body and $\mathbf{X} \in$ $\Omega$ be a material point. Existence of an order parameter function $\eta: \Omega \times(0, T) \rightarrow[0,1]$, where $(0, T) \subset \mathbb{R}$ is a time interval, is assumed. The order parameter function distinguishes between two distinct phases: (i) the original crystal (the parent) and (ii) the twin. Interfaces between phases represent twin boundaries. Order parameter $\eta$ exhibits the following values:

$$
\eta(\mathbf{X}, \bullet)= \begin{cases}0 & \text { if } \mathbf{X} \in \text { parent } \\ (0,1) & \text { if } \mathbf{X} \in \text { twin boundary } \\ 1 & \text { if } \mathbf{X} \in \text { twin. }\end{cases}
$$

According to diffuse interface theory [1,2], $\eta$ is commonly presumed at least $C^{2}$ continuous with respect to $\mathbf{X}$.

\subsection{Kinematics}

A motion of $\Omega$ on $(0, T) \subset \mathbb{R}$ is given by a map $\chi: \Omega \times(0, T) \rightarrow$ $\mathbb{R}^{3}$. Spatial coordinates $\mathbf{x}$ and reference coordinates $\mathbf{X} \in \Omega$ of a 
material particle are thus related by

$\mathbf{x}=\chi(\mathbf{X}, t)$.

The deformation gradient is

$\mathbf{F}=\nabla \chi, \quad F_{a A}=\nabla_{A} \chi_{a}$,

with $\nabla_{A}=\partial / \partial X_{A}$ the material gradient. In regions of the body where $\chi$ is at least $C^{2}$ with respect to $\mathbf{X}$, associated compatibility conditions are $\nabla \times \mathbf{F}=\nabla \times(\nabla \chi)=\mathbf{0}$ where $\times$ is the vector cross product. Associated with twinning kinematics is the simple shear [10]

$\hat{\mathbf{F}}=\mathbf{1}+\gamma_{0} \mathbf{s} \otimes \mathbf{m} \quad$ (with $\mathbf{s} \cdot \mathbf{m}=0$ and $\left.\mathbf{s} \cdot \mathbf{s}=\mathbf{m} \cdot \mathbf{m}=1\right)$.

The unit tensor is $\mathbf{1}$. The unit normal to the surface of composition (i.e., the habit plane) in the reference configuration is $\mathbf{m}$. The magnitude of twinning shear and shear direction are $\gamma_{0}$ and $\mathbf{s}$, respectively; these are both constants in a homogeneous crystal. Let $\mathbf{F}^{+}$and $\mathbf{F}^{-}$denote deformation gradients in twin and parent, respectively, both in their null strain energy reference states. The difference between these deformation gradients is the rank-one matrix

$\mathbf{F}^{+}-\mathbf{F}^{-}=\hat{\mathbf{F}}-\mathbf{1}=\gamma_{0} \mathbf{s} \otimes \mathbf{m}$.

An interpolation procedure [20] is used to characterize the twinning shear in the interfacial regions. Deformation gradient (3) is decomposed multiplicatively as

$\mathbf{F}=\mathbf{F}^{E} \mathbf{F}^{\eta}$

where

$\mathbf{F}^{E}(\mathbf{X}, t)=\mathbf{F}\left(\mathbf{F}^{\eta}\right)^{-1} \equiv$ elastic deformation,

$\mathbf{F}^{\eta}[\eta(\mathbf{X}, t)] \equiv$ stress-free twinning shear.

Superscripts $E$ and $\eta$ are descriptive labels and not numerical exponents. Specifically, twinning shear is interpolated in interfacial regions as follows:

$\mathbf{F}^{\eta}=\mathbf{1}+[\varphi(\eta)] \gamma_{0} \mathbf{s} \otimes \mathbf{m}$,

where $\varphi:[0,1] \rightarrow[0,1]$ is a monotonically increasing function obeying $\varphi(0)=0$ and $\varphi(1)=1$. A representative function also satisfying the condition of vanishing derivative at the endpoints $\left[\varphi^{\prime}(0)=\varphi^{\prime}(1)=0\right.$ ] that will be used later is a "2-3-4 polynomial" [35].

$\varphi(\eta)=\alpha \eta^{2}+2(2-\alpha) \eta^{3}+(\alpha-3) \eta^{4}$,

where $\alpha$ is a scalar constant within the limits $0<\alpha<6$. It follows that in the twin, $\mathbf{F}^{\eta}(1)=\mathbf{1}+\gamma_{0} \mathbf{s} \otimes \mathbf{m}=\hat{\mathbf{F}}$, and in the parent, $\mathbf{F}^{\eta}(0)=\mathbf{1}$. Twinning preserves the volume and mass density of a material element of the solid: $\operatorname{det} \mathbf{F}^{\eta}=\operatorname{det}(\mathbf{1}+$ $\left.\varphi \gamma_{0} \mathbf{s} \otimes \mathbf{m}\right)=1+\varphi \gamma_{0} \mathbf{s} \cdot \mathbf{m}=1$. Fig. 1 shows an elastic body undergoing twin nucleation. The intermediate configuration, twinned but elastically unloaded, is labeled "fictitious" as a result of incompatibility conditions $\nabla \times \mathbf{F}^{\eta} \neq \mathbf{0}$ [36]. While the present theory is fully three-dimensional, a two-dimensional coordinate system is inscribed on the reference body for later reference in Section 5 . The theory presented here does not address plastic slip, i.e., glide of full and/or partial dislocation lines and loops not associated with twinning. Additional model features are required to simultaneously address plastic slip and twinning $[16,36]$.

\subsection{Free energy functional}

The total free energy functional for a body undergoing twinning deformation is written as

$\Psi(\chi, \eta)=\int_{\Omega} W(\mathbf{X}, \mathbf{F}, \eta) \mathrm{d} \Omega+\int_{\Omega} f(\mathbf{X}, \eta, \nabla \eta) \mathrm{d} \Omega$,

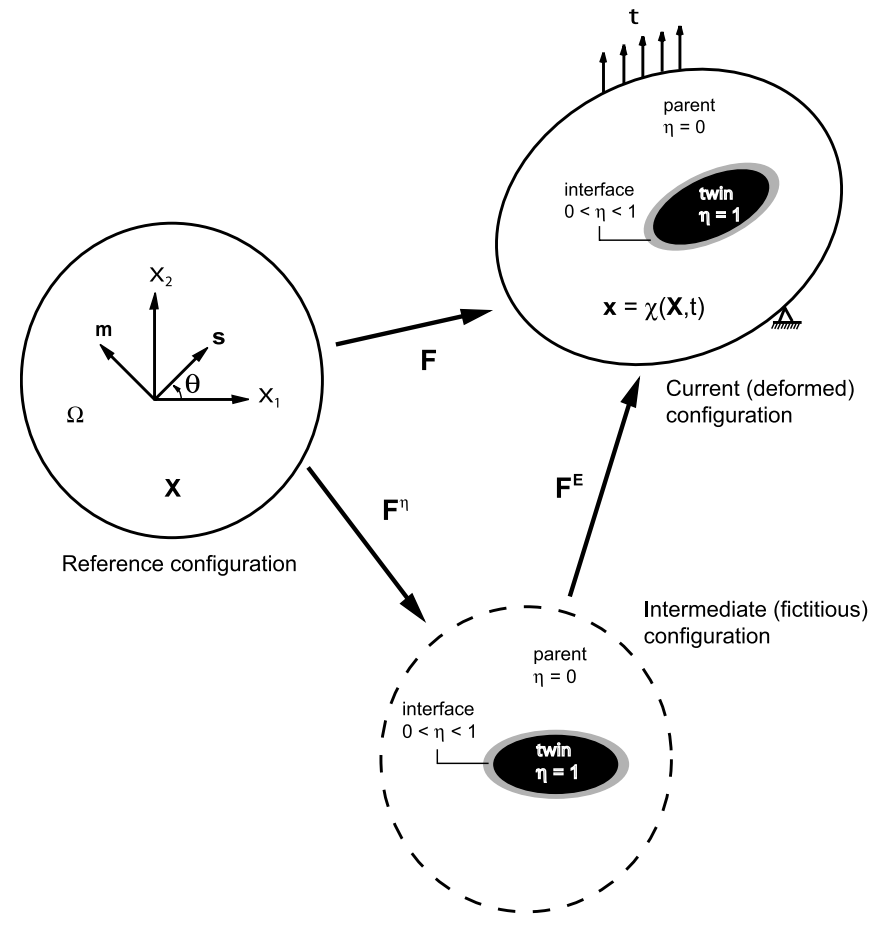

Fig. 1. Phases and kinematics of elasticity and twinning.

where $W: \Omega \times \mathbb{R}_{+}^{3 \times 3} \times[0,1] \rightarrow \mathbb{R}$ is the elastic strain energy density (generally nonzero in parent, twin, and interfacial regions), and where $f: \Omega \times[0,1] \times \mathbb{R}^{3} \rightarrow \mathbb{R}$ accounts for interfacial energy in twin boundary regions. $\mathbb{R}_{+}^{3 \times 3}$ denotes the set of $3 \times 3$ matrices with positive determinant. Henceforth, the following functional form of the strain energy per unit reference volume is used:

$W(\mathbf{X}, \mathbf{F}, \eta)=W\left[\mathbf{E}^{E}(\mathbf{F}, \eta), \eta\right]$,

$\mathbf{E}^{E}=\frac{1}{2}\left(\mathbf{C}^{E}-\mathbf{1}\right)=\frac{1}{2}\left(\mathbf{F}^{E^{T}} \mathbf{F}^{E}-\mathbf{1}\right)$,

with $\mathbf{C}^{E}$ the elastic deformation tensor and $\mathbf{E}^{E}$ the elastic strain tensor. The elastic strain can be expressed in terms of $\mathbf{F}$ and $\eta$ via use of (7) and (8). For any value of order parameter $\eta$, strain energy density vanishes at null elastic strain:

$W(\mathbf{0}, \bullet)=0$.

A quadratic form for the strain energy density is assumed. Higher-order elastic coefficients [16,20,37] can be incorporated by straightforward extension. Dependence of $W\left(\mathbf{E}^{E}, \eta\right)$ on $\eta$ manifests explicitly only via anisotropic elastic coefficients. Strain energy density and second-order moduli are written

$W=\frac{1}{2} \mathbf{E}^{E}: \mathbb{C}(\eta): \mathbf{E}^{E}, \quad \mathbb{C}(\eta)=\left.\frac{\partial^{2} W}{\partial \mathbf{E}^{E} \partial \mathbf{E}^{E}}\right|_{\mathbf{E}^{E}=\mathbf{0}}$

As usual, indices $C_{A B C D}=C_{B A D C}=C_{C D A B}$ and the Laue group of the crystal dictates any other symmetries of $\mathbb{C}(0)$ in the crystallographic frame [37]. For a compound twin in a centrosymmetric structure, re-orientation matrix $\mathbf{Q}$ associated with twinning is [11]

$\mathbf{Q}=2 \mathbf{m} \otimes \mathbf{m}-\mathbf{1}$.

Elastic coefficients of the fully twinned crystal are related to those of the parent by

$C_{A B C D}(1)=Q_{A E} Q_{B F} Q_{C G} Q_{D H} C_{E F G H}(0)$.

Elastic coefficients in interfacial regions are interpolated the same way as the twinning shear:

$\mathbb{C}(\eta)=\mathbb{C}(0)+[\mathbb{C}(1)-\mathbb{C}(0)] \varphi(\eta)$, 
where $\varphi(\eta)$ obeys (9). Different interpolators could be used for twinning shear and elastic coefficients; for simplicity, the same function is prescribed here for both. In the isotropic elastic approximation, letting $\lambda$ denote Lamé's constant and $\mu$ the shear modulus, the elasticity tensor

$C_{A B C D}=\lambda \delta_{A B} \delta_{C D}+\mu\left(\delta_{A C} \delta_{B D}+\delta_{A D} \delta_{B C}\right)$,

so that $\mathbb{C}(0)=\mathbb{C}(1)$ and $W\left(\mathbf{E}^{E}, \eta\right) \rightarrow W\left(\mathbf{E}^{E}\right)$ does not explicitly depend on $\eta$.

The local interfacial energy per unit reference volume follows from the Cahn-Hilliard formalism [1]:

$f(\eta, \nabla \eta)=f_{0}(\eta)+\kappa:(\nabla \eta \otimes \nabla \eta)$,

with $\kappa$ a symmetric second-order tensor that may generally depend on $\eta$, but is assumed here for simplicity to have constant components. When interfacial energy is isotropic, $\kappa=\kappa \mathbf{1}$ and

$f(\eta, \nabla \eta)=f_{0}(\eta)+\kappa|\nabla \eta|^{2}$.

Prescribed for $f_{0}$ is a standard "double-well" potential [5,7,20,35]:

$f_{0}(\eta)=A \eta^{2}(1-\eta)^{2}$,

with $A>0$. As shown in Appendix C, in the isotropic approximation $A$ and $\kappa$ are related to equilibrium energy per unit area $\Gamma$ and thickness $l$ of an unstressed interface via

$\kappa=3 \Gamma l / 4, \quad A=12 \Gamma / l$.

The total free energy functional $\Psi$ of (10) becomes, using (13) and (18),

$$
\begin{aligned}
\Psi(\chi, \eta)= & \frac{1}{2} \int_{\Omega} \mathbf{E}^{E}: \mathbb{C}(\eta): \mathbf{E}^{E} \mathrm{~d} \Omega \\
& +\int_{\Omega}\left[A \eta^{2}(1-\eta)^{2}+\kappa:(\nabla \eta \otimes \nabla \eta)\right] \mathrm{d} \Omega .
\end{aligned}
$$

For isotropic elastic and interfacial energies, with $\operatorname{tr} \mathbf{A}=A_{A A}$ the trace of a second-order tensor,

$$
\begin{aligned}
\Psi(\chi, \eta)= & \int_{\Omega}\left[(\lambda / 2)\left(\operatorname{tr} \mathbf{E}^{E}\right)^{2}+\mu \mathbf{E}^{E}: \mathbf{E}^{E}\right] \mathrm{d} \Omega \\
& +\int_{\Omega}\left[A \eta^{2}(1-\eta)^{2}+\kappa|\nabla \eta|^{2}\right] \mathrm{d} \Omega .
\end{aligned}
$$

\subsection{Equilibrium conditions}

A stable configuration of a body undergoing twinning deformation corresponds to a minimizer of total free energy functional (10), given certain boundary conditions. The problem of finding such stable configuration can be expressed as

$\min _{\chi, \eta} \Psi(\chi, \eta)$.

Substantial literature $[38,39]$ has been dedicated to assessment of existence of minimizers such as in (24). In general, such minimizers need not exist for arbitrary energy density functions $f$ and $W$. Moreover, existence requirements impose constraints on functional forms of $f$ and $W$. In Appendix A, existence of minimizers of (24) is explored under assumptions of convexity or polyconvexity of strain energy density function $W$ and interfacial energy density function $f[40,39]$.

The following variational equation is posited that will suggest, upon application of Hamilton's principle, both weak and strong forms of static equilibrium equations and boundary conditions:

$\delta \Psi-\int_{\partial \Omega} \mathbf{t} \cdot \delta \chi \mathrm{d} S-\int_{\partial \Omega} h \delta \eta \mathrm{d} S=0$,

where $\mathbf{t}$ is a mechanical traction vector per unit reference area, $\partial \Omega$ is the boundary of $\Omega, \mathrm{d} S$ is a surface element of $\partial \Omega$, and $h$ is a scalar conjugate force (energy per unit reference area) to variations of the order parameter. Assuming $C^{1}$-smoothness of $f$ and taking the first variation of the interfacial energy,

$\delta \int_{\Omega} f \mathrm{~d} \Omega=\int_{\Omega} f_{0}^{\prime} \delta \eta \mathrm{d} \Omega+\int_{\Omega} 2 \kappa:(\nabla \eta \otimes \nabla \delta \eta) \mathrm{d} \Omega$,

where $\delta \eta$ denotes an admissible variation of order parameter $\eta$. Similarly, for $W$ a $C^{1}$-function of all its arguments, the first variation of the strain energy is

$\delta \int_{\Omega} W \mathrm{~d} \Omega=\int_{\Omega} \frac{\partial W}{\partial \eta} \delta \eta \mathrm{d} \Omega+\int_{\Omega} \frac{\partial W}{\partial \mathbf{F}}: \nabla \delta \chi \mathrm{d} \Omega$,

with $\delta \chi$ denoting an admissible variation of deformation map $\chi$. Combining (25)-(27) yields the weak form of the equilibrium equations:

$$
\begin{aligned}
& \int_{\Omega}\left(f_{0}^{\prime}+\frac{\partial W}{\partial \eta}\right) \delta \eta \mathrm{d} \Omega+\int_{\Omega} 2 \kappa:(\nabla \eta \otimes \nabla \delta \eta) \mathrm{d} \Omega \\
& \quad+\int_{\Omega} \frac{\partial W}{\partial \mathbf{F}}: \nabla \delta \chi \mathrm{d} \Omega-\int_{\partial \Omega} \mathbf{t} \cdot \delta \chi \mathrm{d} S-\int_{\partial \Omega} h \delta \eta \mathrm{d} S=0
\end{aligned}
$$

for any admissible variations $\delta \chi$ and $\delta \eta$.

Strong forms of equilibrium equations require $C^{2}$-smoothness of both $\chi$ and $\eta$. Application of the divergence theorem to (26) leads to

$$
\begin{aligned}
\delta \int_{\Omega} f \mathrm{~d} \Omega= & \int_{\Omega} f_{0}^{\prime} \delta \eta \mathrm{d} \Omega-\int_{\Omega} 2 \kappa:[\nabla(\nabla \eta)] \delta \eta \mathrm{d} \Omega \\
& +\int_{\partial \Omega} 2 \kappa:[(\nabla \eta) \otimes \mathbf{n}] \delta \eta \mathrm{d} S,
\end{aligned}
$$

with $\mathbf{n}$ the unit outward normal to $\partial \Omega$. The first variation of the strain energy can be converted analogously into

$$
\begin{aligned}
\delta \int_{\Omega} W \mathrm{~d} \Omega= & \int_{\Omega} \frac{\partial W}{\partial \eta} \delta \eta \mathrm{d} \Omega-\int_{\Omega}\left[\nabla \cdot \frac{\partial W}{\partial \mathbf{F}}\right] \cdot \delta \chi \mathrm{d} \Omega \\
& +\int_{\partial \Omega}\left[\mathbf{n} \cdot \frac{\partial W}{\partial \mathbf{F}}\right] \cdot \delta \chi \mathrm{d} S .
\end{aligned}
$$

Local strong forms of equilibrium equations (Euler-Lagrange equations) follow directly from (29) and (30):

$\left.\nabla \cdot \frac{\partial W}{\partial \mathbf{F}}\right|_{\eta}=\nabla \cdot \mathbf{P}=0,\left.\quad \nabla_{A} \frac{\partial W}{\partial \nabla_{A} \chi_{a}}\right|_{\eta}=\nabla_{A} P_{a A}=0$,

$f_{0}^{\prime}-2 \kappa:[\nabla(\nabla \eta)]+\left.\frac{\partial W}{\partial \eta}\right|_{\mathbf{F}}=0$,

$f_{0}^{\prime}-2 \kappa_{A B} \nabla_{A} \nabla_{B} \eta+\left.\frac{\partial W}{\partial \eta}\right|_{\mathbf{F}}=0$

where $\mathbf{P}$ is the first Piola-Kirchhoff stress tensor. Corresponding boundary conditions are

$\mathbf{t}=\mathbf{P n}, \quad t_{a}=P_{a A} n_{A}$,

$h=2 \kappa:(\nabla \eta \otimes \mathbf{n}), \quad h=2 \kappa_{A B} n_{B} \nabla_{A} \eta$.

The first Piola-Kirchhoff stress also obeys

$\mathbf{P}=\left.\frac{\partial W}{\partial \mathbf{F}}\right|_{\eta}=\frac{\partial W}{\partial \mathbf{E}^{E}}: \frac{\partial \mathbf{E}^{E}}{\partial \mathbf{F}^{E}}:\left.\frac{\partial \mathbf{F}^{E}}{\partial \mathbf{F}}\right|_{\eta}=\mathbf{F}^{E} \boldsymbol{\Sigma}\left(\mathbf{F}^{\eta}\right)^{-T}$.

The elastic second Piola-Kirchhoff stress is

$\boldsymbol{\Sigma}=\frac{\partial W}{\partial \mathbf{E}^{E}}=\mathbb{C}: \mathbf{E}^{E}$.

The partial derivative of $W\left[\mathbf{E}^{E}(\mathbf{F}, \eta), \eta\right]$ with $\mathbf{F}$ fixed is computed as

$\left.\frac{\partial W}{\partial \eta}\right|_{\mathbf{F}}=\left.\frac{\partial W}{\partial \eta}\right|_{\mathbf{E}^{E}}+\left.\frac{\partial W}{\partial \mathbf{E}^{E}}\right|_{\eta}:\left.\frac{\partial \mathbf{E}^{E}}{\partial \eta}\right|_{\mathbf{F}}$. 
From (13) and (16),

$$
\begin{aligned}
\left.\frac{\partial W}{\partial \eta}\right|_{\mathbf{E}^{E}} & =\frac{1}{2} \mathbf{E}^{E}: \frac{\partial \mathbb{C}}{\partial \eta}: \mathbf{E}^{E} \\
& =\frac{1}{2} \frac{\partial \varphi}{\partial \eta} \mathbf{E}^{E}:[\mathbb{C}(1)-\mathbb{C}(0)]: \mathbf{E}^{E} .
\end{aligned}
$$

From (6)-(8) and (36),

$$
\begin{aligned}
\left.\frac{\partial W}{\partial \mathbf{E}^{E}}\right|_{\eta}:\left.\frac{\partial \mathbf{E}^{E}}{\partial \eta}\right|_{\mathbf{F}} & =-\left\{\boldsymbol{\Sigma}:\left[\mathbf{C}^{E}(\mathbf{s} \otimes \mathbf{m})\left(\mathbf{F}^{\eta}\right)^{-1}\right]\right\} \gamma_{0} \frac{\partial \varphi}{\partial \eta} \\
& =-\tau \gamma_{0} \frac{\partial \varphi}{\partial \eta} .
\end{aligned}
$$

It can be verified that $\tau$ is a resolved shear stress on the habit plane in the direction of twinning shear. Combining (37)-(39), phase equilibrium condition (32) can be rewritten

$f_{0}^{\prime}-2 \kappa:[\nabla(\nabla \eta)]=\left\{\frac{1}{2} \mathbf{E}^{E}:[\mathbb{C}(0)-\mathbb{C}(1)]: \mathbf{E}^{E}+\tau \gamma_{0}\right\} \frac{\partial \varphi}{\partial \eta}$.

In the isotropic approximation, choosing $\varphi$ from (9) and using $f_{0}$ from (20), equilibrium condition (40) becomes

$$
\begin{aligned}
\tau & {\left[\alpha \eta+3(2-\alpha) \eta^{2}+2(\alpha-3) \eta^{3}\right] } \\
& =\frac{1}{\gamma_{0}}\left[A \eta\left(1-3 \eta+2 \eta^{2}\right)-\kappa \nabla^{2} \eta\right] .
\end{aligned}
$$

Both sides of (41) vanish identically in regions of uniform phase where $\eta=0$ or $\eta=1$. For purposes of comparison, a kinetic equation corresponding to (40) in the context of TDGL theory is listed in Appendix D.

\section{Geometric linearization}

In what follows in Section 3, the theory of Section 2 is linearized for small deformations. The linearized theory proves useful for comparison of numerical results with analytical studies of energetics of twin nucleation [30-33] in the context of Eshelby's model of elastic inclusions and inhomogeneities [41,42]. Eq. (1) still applies.

\subsection{Kinematics}

In linear elasticity, the usual kinematic field variables are displacement $\mathbf{u}(\mathbf{X}, t)=\mathbf{X}(\mathbf{X}, t)-\mathbf{X}$ and its gradient

$\boldsymbol{\beta}=\nabla \mathbf{u}, \quad \beta_{A B}=\nabla_{B} u_{A}$.

In contrast to the geometrically nonlinear theory (Fig. 1), there is no explicit distinction among configurations of a deformable body. Compatibility conditions are $\nabla \times \boldsymbol{\beta}=\nabla \times(\nabla \mathbf{u})=\mathbf{0}$. Decomposition (6) is replaced with

$\boldsymbol{\beta}=\boldsymbol{\beta}^{E}+\boldsymbol{\beta}^{\eta}$,

where $\boldsymbol{\beta}^{E}$ is the elastic distortion and $\boldsymbol{\beta}^{\eta}$ is the distortion associated with twinning shear:

$\boldsymbol{\beta}^{\eta}=[\varphi(\eta)] \gamma_{0} \mathbf{s} \otimes \mathbf{m}$.

The symmetric elastic strain tensor is

$$
\begin{aligned}
\boldsymbol{\varepsilon}^{E}(\nabla \mathbf{u}, \eta) & =\frac{1}{2}\left\{\boldsymbol{\beta}^{E}+\boldsymbol{\beta}^{E^{T}}\right\} \\
& =\frac{1}{2}\left\{\nabla \mathbf{u}+(\nabla \mathbf{u})^{T}-\gamma_{0}[\varphi(\eta)][\mathbf{s} \otimes \mathbf{m}+\mathbf{m} \otimes \mathbf{s}]\right\} .
\end{aligned}
$$

\subsection{Free energy}

The total free energy functional for a body of reference volume $\Omega$ is written as

$\Psi(\mathbf{u}, \eta)=\int_{\Omega} W(\nabla \mathbf{u}, \eta) \mathrm{d} \Omega+\int_{\Omega} f(\eta, \nabla \eta) \mathrm{d} \Omega$, where $W$ is the elastic strain energy density and $f$ accounts for interfacial energy. Strain energy density and second-order elastic moduli are

$$
\begin{gathered}
W=W\left[\boldsymbol{\varepsilon}^{E}(\nabla \mathbf{u}, \eta), \eta\right]=\frac{1}{2} \boldsymbol{\varepsilon}^{E}: \mathbb{C}(\eta): \boldsymbol{\varepsilon}^{E}, \\
\mathbb{C}(\eta)=\left.\frac{\partial^{2} W}{\partial \boldsymbol{\varepsilon}^{E} \partial \boldsymbol{\varepsilon}^{E}}\right|_{\boldsymbol{\varepsilon}^{E}=0} .
\end{gathered}
$$

Eqs. (14)-(21) apply in the linearized case. The total free energy functional $\Psi$ of (46) becomes, using (18) and (47),

$$
\begin{aligned}
\Psi(\mathbf{u}, \eta)= & \frac{1}{2} \int_{\Omega} \boldsymbol{\varepsilon}^{E}: \mathbb{C}(\eta): \boldsymbol{\varepsilon}^{E} \mathrm{~d} \Omega \\
& +\int_{\Omega}\left[A \eta^{2}(1-\eta)^{2}+\kappa:(\nabla \eta \otimes \nabla \eta)\right] \mathrm{d} \Omega .
\end{aligned}
$$

For isotropic elastic and interfacial energies, this reduces to

$$
\begin{aligned}
\Psi(\mathbf{u}, \eta)= & \int_{\Omega}\left[(\lambda / 2)(\operatorname{tr} \nabla \mathbf{u})^{2}+\mu\left(\nabla \mathbf{u}-\gamma_{0} \varphi \mathbf{s} \otimes \mathbf{m}\right)_{\text {symm }}\right. \\
& \left.:\left(\nabla \mathbf{u}-\gamma_{0} \varphi \mathbf{s} \otimes \mathbf{m}\right)_{\text {symm }}\right] \mathrm{d} \Omega \\
& +\int_{\Omega}\left[A \eta^{2}(1-\eta)^{2}+\kappa|\nabla \eta|^{2}\right] \mathrm{d} \Omega,
\end{aligned}
$$

where $(\bullet)_{\text {symm }}$ denotes the symmetric part of a second-order tensor, e.g., $2 \mathbf{A}_{\text {symm }}=\mathbf{A}+\mathbf{A}^{T}$.

\subsection{Equilibrium conditions}

The following variational equation is posited that will suggest, upon application of Hamilton's principle, local or strong forms of static equilibrium equations and boundary conditions:

$\delta \Psi-\int_{\partial \Omega} \mathbf{t} \cdot \delta \mathbf{u} \mathrm{d} S-\int_{\partial \Omega} h \delta \eta \mathrm{d} S=0$,

where $\mathbf{t}$ is a mechanical traction vector per unit area, $\mathrm{d} S$ is a surface element of $\partial \Omega$, and $h$ is a scalar conjugate force to variations of the order parameter. Taking the first variation of the interfacial energy and applying the divergence theorem gives (29). The first variation of the strain energy with $\nabla \mathbf{u}$ and $\eta$ independent is

$$
\begin{aligned}
\delta \int_{\Omega} W \mathrm{~d} \Omega= & \int_{\Omega} \frac{\partial W}{\partial \eta} \delta \eta \mathrm{d} \Omega-\int_{\Omega}\left[\nabla \cdot \frac{\partial W}{\partial \nabla \mathbf{u}}\right] \cdot \delta \mathbf{u} \mathrm{d} \Omega \\
& +\int_{\partial \Omega}\left[\mathbf{n} \cdot \frac{\partial W}{\partial \nabla \mathbf{u}}\right] \cdot \delta \mathbf{u} \mathrm{d} S .
\end{aligned}
$$

It follows from (29) and (51) that in $\Omega$, the Euler-Lagrange equations are

$\left.\nabla \cdot \frac{\partial W}{\partial \nabla \mathbf{u}}\right|_{\eta}=\nabla \cdot \sigma=0$,

$f_{0}^{\prime}-2 \boldsymbol{\kappa}:[\nabla(\nabla \eta)]+\left.\frac{\partial W}{\partial \eta}\right|_{\nabla \eta}=0$,

where $\sigma$ is the symmetric stress tensor. Corresponding boundary conditions are

$\mathbf{t}=\boldsymbol{\sigma} \mathbf{n}, \quad h=2 \boldsymbol{\kappa}:(\nabla \eta \otimes \mathbf{n})$.

The stress also obeys, from (47),

$\boldsymbol{\sigma}=\left.\frac{\partial W}{\partial \nabla \mathbf{u}}\right|_{\eta}=\frac{\partial W}{\partial \boldsymbol{\varepsilon}^{E}}:\left.\frac{\partial \boldsymbol{\varepsilon}^{E}}{\partial \nabla \mathbf{u}}\right|_{\eta}=\frac{\partial W}{\partial \boldsymbol{\varepsilon}^{E}}=\mathbb{C}: \boldsymbol{\varepsilon}^{E}$

The partial derivative of $W\left[\varepsilon^{E}(\nabla \mathbf{u}, \eta), \eta\right]$ with $\nabla \mathbf{u}$ fixed is

$$
\left.\frac{\partial W}{\partial \eta}\right|_{\nabla \mathbf{u}}=\left.\frac{\partial W}{\partial \eta}\right|_{\boldsymbol{\varepsilon}^{E}}+\left.\frac{\partial W}{\partial \boldsymbol{\varepsilon}^{E}}\right|_{\eta}:\left.\frac{\partial \boldsymbol{\varepsilon}^{E}}{\partial \eta}\right|_{\nabla \mathbf{u}} .
$$


From (16) and (47),

$\left.\frac{\partial W}{\partial \eta}\right|_{\boldsymbol{\varepsilon}^{E}}=\frac{1}{2} \boldsymbol{\varepsilon}^{E}: \frac{\partial \mathbb{C}}{\partial \eta}: \boldsymbol{\varepsilon}^{E}=\frac{1}{2} \frac{\partial \varphi}{\partial \eta} \boldsymbol{\varepsilon}^{E}:[\mathbb{C}(1)-\mathbb{C}(0)]: \boldsymbol{\varepsilon}^{E}$.

From (42)-(45) and (54),

$\left.\frac{\partial W}{\partial \boldsymbol{\varepsilon}^{E}}\right|_{\eta}:\left.\frac{\partial \boldsymbol{\varepsilon}^{E}}{\partial \eta}\right|_{\nabla \mathbf{u}}=-\{\boldsymbol{\sigma}:(\mathbf{s} \otimes \mathbf{m})\} \gamma_{0} \frac{\partial \varphi}{\partial \eta}=-\tau \gamma_{0} \frac{\partial \varphi}{\partial \eta}$,

where $\tau$ is a resolved shear stress acting on the habit plane in the direction of twinning shear. Combining (55)-(57), the second of equilibrium conditions (52) can be rewritten

$f_{0}^{\prime}-2 \kappa:[\nabla(\nabla \eta)]=\left\{\frac{1}{2} \boldsymbol{\varepsilon}^{E}:[\mathbb{C}(0)-\mathbb{C}(1)]: \boldsymbol{\varepsilon}^{E}+\tau \gamma_{0}\right\} \frac{\partial \varphi}{\partial \eta}$.

In the isotropic approximation, choosing $\varphi$ from (9) and $f_{0}$ from (20), (58) reduces to (41), but with $\tau$ defined in (57) rather than (39). When elastic strains are small, $\Sigma \approx \sigma, \mathbf{C}^{E} \approx \mathbf{1}$, and the difference between $\tau$ in (39) and (57) is on the order of twinning shear $\gamma_{0}$.

\section{Numerical methods}

The finite element method is used to seek solutions for equilibrium or local minimum energy states of a body subjected to boundary conditions. At each reference point $\mathbf{X}$, primary solution variables are displacement $\mathbf{u}$ and order parameter $\eta$. If these solution variables and requisite material properties are known, then all elastic field variables and interfacial quantities can be computed via the appropriate mathematical operations given in Sections 2 and 3.

\subsection{Finite element discretization}

The body of reference volume $\Omega$ is discretized into a number of standard finite elements with shape functions $N_{i}(\mathbf{X})$. Let $\mathbf{u}_{i}(t)$ and $\eta_{i}(t)$ denote instantaneous values of displacement and order parameter, respectively, at node $i$. Displacement and order parameter fields are represented in a finite element context as, respectively,

$\mathbf{u}(\mathbf{X}, t)=\mathbf{u}_{i}(t) N_{i}(\mathbf{X}), \quad \eta(\mathbf{X}, t)=\eta_{i}(t) N_{i}(\mathbf{X})$,

where summation proceeds over all nodes $i$; however, only those nodes supporting the element(s) containing or bounding point $\mathbf{X}$ have $N_{i}(\mathbf{X}) \neq 0$. Material gradients of (59) follow as

$\mathbf{F}=\mathbf{1}+\nabla \mathbf{u}=\mathbf{1}+\mathbf{u}_{i} \otimes \nabla N_{i}, \quad \nabla \eta=\eta_{i} \nabla N_{i}$.

\subsection{Energy minimization}

The finite element method is employed to seek solutions of weak forms of equilibrium conditions (28). Strong forms derived in Sections 2.4 and 3.3 are not needed by the numerical algorithms. Addressed in what follows are the following kinds of boundary conditions:

$\partial \Omega=\partial \Omega_{M} \cap \partial \Omega_{P}$

$\partial \Omega_{M}=\partial \Omega_{M, D} \cup \partial \Omega_{M, N}, \emptyset=\partial \Omega_{M, D} \cap \partial \Omega_{M, N}$

$\mathbf{u}(\mathbf{X}, t)$ prescribed on $\partial \Omega_{M, D}, \mathbf{t}(\mathbf{X}, t)=0$ on $\left.\partial \Omega_{M, N}\right\}$

mechanical conditions

$\partial \Omega_{P}=\partial \Omega_{P, D} \cup \partial \Omega_{P, N}, \varnothing=\partial \Omega_{P, D} \cap \partial \Omega_{P, N}$

$\eta(\mathbf{X}, t)$ prescribed on $\partial \Omega_{P, D}, h(\mathbf{X}, t)=0$ on $\left.\partial \Omega_{P, N}\right\}$

phase field conditions

Dirichlet conditions for displacement and the order parameter are applied on $\partial \Omega_{M, D}$ and $\partial \Omega_{P, D}$, respectively. Neumann conditions corresponding to free surfaces are applied on $\partial \Omega_{M, N}$ and $\partial \Omega_{P, N}$, respectively. The present treatment can easily be extended to address arbitrary Neumann conditions if terms accounting for external work are incorporated in the energy functional whose stationary points are sought.

First consider the geometrically nonlinear theory of Section 2. The free energy functional is (22) in the general anisotropic case. Nodal equilibrium conditions are obtained by substituting (59) and (60) into (22) and differentiating with respect to nodal degrees of freedom:

$$
\begin{aligned}
\mathbf{0}= & \frac{\partial \Psi}{\partial \mathbf{u}_{i}}=\int_{\Omega} \frac{\partial W}{\partial \mathbf{F}}: \frac{\partial \mathbf{F}}{\partial \mathbf{u}_{i}} \mathrm{~d} \Omega=\int_{\Omega} \mathbf{P} \nabla N_{i} \mathrm{~d} \Omega \\
= & \int_{\Omega}\left[\mathbf{F}^{E}\left(\mathbb{C}: \mathbf{E}^{E}\right)\left(\mathbf{F}^{\eta}\right)^{-T}\right] \nabla N_{i} \mathrm{~d} \Omega, \\
0= & \frac{\partial \Psi}{\partial \eta_{i}} \\
= & \int_{\Omega} \frac{\partial f}{\partial \eta} N_{i} \mathrm{~d} \Omega+\int_{\Omega} \frac{\partial f}{\partial \nabla \eta} \cdot \nabla N_{i} \mathrm{~d} \Omega+\int_{\Omega} \frac{\partial W}{\partial \eta} N_{i} \mathrm{~d} \Omega \\
= & \int_{\Omega}\left\{2 A \eta\left(1-3 \eta+2 \eta^{2}\right)\right\} N_{i} \mathrm{~d} \Omega+\int_{\Omega}(2 \kappa \nabla \eta) \cdot \nabla N_{i} \mathrm{~d} \Omega \\
& +\int_{\Omega}\left\{\frac{1}{2} \mathbf{E}^{E}:[\mathbb{C}(1)-\mathbb{C}(0)]: \mathbf{E}^{E}-\tau \gamma_{0}\right\} \frac{\partial \varphi}{\partial \eta} N_{i} \mathrm{~d} \Omega .
\end{aligned}
$$

In general, solutions of (62) and (63), corresponding to stationary points $\delta \Psi\left(\mathbf{u}_{i}, \eta_{i}\right)=0$, can be associated with local energy minima, maxima, or saddle points. Energy functional $\Psi\left(\mathbf{u}_{i}, \eta_{i}\right)$ is not necessarily convex in its arguments, and may exhibit multiple local minima, for example [20]. A conjugate gradient algorithm is used to seek minimum energy states corresponding to (62) and (63). Initial conditions are also prescribed as part of the solution procedure. For example, in Section 5 an initial twin nucleus $(\eta=1)$ is placed within a larger domain wherein $\eta=0$ initially. Such a system will not be in mechanical or phase equilibrium at the initial time.

Now consider the linearized model of Section 3, with energy functional (48). Global discretized equilibrium conditions are

$$
\begin{aligned}
\mathbf{0}= & \frac{\partial \Psi}{\partial \mathbf{u}_{i}}=\int_{\Omega} \frac{\partial W}{\partial \nabla \mathbf{u}}: \frac{\partial \nabla \mathbf{u}}{\partial \mathbf{u}_{i}} \mathrm{~d} \Omega=\int_{\Omega} \sigma \nabla N_{i} \mathrm{~d} \Omega \\
= & \int_{\Omega}\left(\mathbb{C}: \boldsymbol{\varepsilon}^{E}\right) \nabla N_{i} \mathrm{~d} \Omega, \\
0= & \frac{\partial \Psi}{\partial \eta_{i}}=\int_{\Omega}\left[2 A \eta\left(1-3 \eta+2 \eta^{2}\right)\right] N_{i} \mathrm{~d} \Omega \\
& +\int_{\Omega}(2 \kappa \nabla \eta) \cdot \nabla N_{i} \mathrm{~d} \Omega \\
& +\int_{\Omega}\left\{\frac{1}{2} \boldsymbol{\varepsilon}^{E}:[\mathbb{C}(1)-\mathbb{C}(0)]: \boldsymbol{\varepsilon}^{E}-\tau \gamma_{0}\right\} \frac{\partial \varphi}{\partial \eta} N_{i} \mathrm{~d} \Omega .
\end{aligned}
$$

For the isotropic case (49) with interpolator $\varphi$ from (9), these reduce to

$$
\begin{aligned}
\mathbf{0}= & \frac{\partial \Psi}{\partial \mathbf{u}_{i}} \\
= & \int_{\Omega}\left\{\lambda(\nabla \cdot \mathbf{u}) \mathbf{1}+2 \mu\left[\nabla \mathbf{u}-\gamma_{0}\left[\alpha \eta^{2}+2(2-\alpha) \eta^{3}\right.\right.\right. \\
& \left.\left.\left.+(\alpha-3) \eta^{4}\right] \mathbf{s} \otimes \mathbf{m}\right]_{s y m m}\right\} \nabla N_{i} \mathrm{~d} \Omega \\
0 & \frac{\partial \Psi}{\partial \eta_{i}} \\
= & \int_{\Omega}\left\{2 A \eta\left(1-3 \eta+2 \eta^{2}\right)\right\} N_{i} \mathrm{~d} \Omega+\int_{\Omega}\{2 \kappa \nabla \eta\} \cdot \nabla N_{i} \mathrm{~d} \Omega \\
& +\int_{\Omega}\left\{\mu \gamma _ { 0 } \left[\left[\alpha \eta^{2}+2(2-\alpha) \eta^{3}+(\alpha-3) \eta^{4}\right] \gamma_{0}\right.\right. \\
& \left.-2 \nabla \mathbf{u}:(\mathbf{s} \otimes \mathbf{m})_{s y m m}\right] \\
& \left.\times\left[2 \alpha \eta+6(2-\alpha) \eta^{2}+4(\alpha-3) \eta^{3}\right]\right\} N_{i} \mathrm{~d} \Omega .
\end{aligned}
$$


Coupling among $\eta, \nabla \eta$, and $\nabla \mathbf{u}$ depicts interaction of the order parameter and its gradient with elastic fields.

\section{Application: twin nucleation in magnesium single crystals}

The phase field theory and numerical methods discussed in Sections 2-4 are applied to the problem of homogeneous twin nucleation.

\subsection{Background: homogeneous twin nucleation}

Homogeneous twin nucleation is defined as the nucleation of a twin embryo within an otherwise perfect single crystal [11]. Analytical models based on free energy variation concepts in the context of phase transformations [42] have been applied to describe twin nucleation [11,30-33,43]. Such analytical approaches consider nucleation of a twin embryo of idealized geometry - an elliptical cylinder in two dimensions or an ellipsoid in three dimensions - embedded in an infinite medium, with a perfectly bonded (i.e., coherent) sharp interface separating inclusion from surrounding medium (i.e., the matrix). The solution technique involves simultaneous solution of two equilibrium equations associated with stationary points of the total (Gibbs) free energy change associated with twinning. These two equations yield the critical size and aspect ratio of the inclusion, as outlined in Appendix E. Apparently, exact solutions are available only for approximations of linear elasticity, isotropic surface energy associated with the twin boundary, and traction boundary conditions at infinity. The solution provides the critical size and shape of an inclusion for a given set of material properties - elastic constants, twin boundary surface energy, and twinning transformation shear - and far-field applied stress. At the critical aspect ratio, the critical size corresponds to unstable equilibrium [31,32], i.e., a saddle point on the free energy surface. At a given far-field applied stress, a twin nucleus smaller than the critical size will tend to shrink and disappear, while one larger than the critical size will tend to grow in an unstable manner. The larger the applied stress component resolved on the habit plane in the twinning direction, the smaller the critical size, meaning that nucleation of a small twin becomes more energetically favorable as the resolved shear stress increases. In a real heterogeneous material at finite temperature, nuclei of various sizes and shapes emerge as a result of local statistical fluctuations $[11,31,32]$. According to the theory, those nuclei larger than the critical size would form twins that grow until interactions with other defects, grain boundaries, or external surfaces occur.

In the present paper, the phase field approach is used to model the problem of homogeneous twin nucleation. Numerical results obtained from the phase field model, under assumptions of geometric linearity (Section 3) and plane strain boundary conditions, are compared with the analytical solution [30]. Such an exercise provides validation for the phase field model and numerical methods advanced here. Additional numerical simulations consider effects of anisotropic twin boundary (surface) energy, variable equilibrium thickness of the twin boundary region, various habit plane directions (i.e., lattice orientations), and various boundary conditions (Dirichlet vs. Neumann, shear vs. tension, and domain shapes). Such additional factors cannot all be addressed via known analytical elasticity solution techniques.

\subsection{Material}

Pure magnesium single crystals are studied. Properties are listed in Table 1 with supporting references. Magnesium exhibits a hexagonal crystal structure and is centrosymmetric, with $c / a=1.6235$. The twinning system of consideration is the primary one: $\langle 10 \overline{1} 1\rangle\{\overline{1} 012\}$ with twinning shear $\gamma_{0}=(3-$ $\left.c^{2} / a^{2}\right) /\left(3^{1 / 2} c / a\right)=0.1295[11,34]$. All five independent secondorder elastic constants [44] are listed in Table 1, though in
Table 1

Properties for pure Mg single crystals.

\begin{tabular}{|c|c|c|c|}
\hline Parameter & Value & Definition & Reference \\
\hline$c$ & $5.200 \AA ̊$ & lattice parameters $(0 \mathrm{~K})$ & [13] \\
\hline$a$ & $3.203 \AA$ & & \\
\hline$\gamma_{0}$ & 0.1295 & shear for $\langle 10 \overline{1} 1\rangle\langle\overline{1} 012\}$ twin & [11] \\
\hline$C_{11}$ & $63.5 \mathrm{GPa}$ & second-order elastic constants; & [44] \\
\hline$C_{33}$ & $66.5 \mathrm{GPa}$ & extrapolated from 4.2 to $0 \mathrm{~K}$ & \\
\hline$C_{12}$ & $25.9 \mathrm{GPa}$ & & \\
\hline$C_{13}$ & $21.7 \mathrm{GPa}$ & & \\
\hline$C_{44}$ & $18.4 \mathrm{GPa}$ & & \\
\hline$\lambda$ & $24.0 \mathrm{GPa}$ & Lamé constant (Voigt average) & \\
\hline$\mu$ & $19.4 \mathrm{GPa}$ & shear modulus (Voigt average) & \\
\hline$K$ & 36.7 GPa & bulk modulus (Voigt average) & \\
\hline$v$ & 0.276 & Poisson's ratio (Voigt average) & \\
\hline$\Gamma$ & $117 \mathrm{~mJ} / \mathrm{m}^{2}$ & twin boundary energy & [13] \\
\hline$l$ & $1.0 \mathrm{~nm}$ & equilibrium boundary thickness & {$[20,45]$} \\
\hline$\kappa$ & $0.0878 \mathrm{~nJ} / \mathrm{m}$ & gradient energy parameter & Eq. (21) \\
\hline$A$ & $1.404 \mathrm{GPa}$ & double-well energy parameter & Eq. (21) \\
\hline
\end{tabular}

calculations that follow, Voigt averages [46] are used for isotropic elastic constants. Magnesium single crystals are not strongly anisotropic elastically [47] [ $C_{11} \approx C_{33}, C_{12} \approx C_{13}$, and $C_{44} \approx\left(C_{11}-\right.$ $\left.C_{12}\right) / 2$ ], so the isotropic elastic approximation appears reasonable. Reuss averages [46] for shear and bulk moduli are 19.3 GPa and 36.7 GPa, respectively, nearly identical to Voigt averages in Table 1.

Twin boundary surface energy is obtained from DFT calculations [13]. In most phase field simulations discussed later, surface energy is assumed isotropic, following usual theoretical studies [30-33], but in one case anisotropy of the surface energy is considered. In a material coordinate system with axes aligned parallel to twinning direction and habit plane normal $\left(X_{1} \| \mathbf{s}\right.$ and $\left.X_{2} \| \mathbf{m}\right)$, the gradient coefficient entering (48) is written

$[\kappa]=\left[\begin{array}{ll}\kappa_{11} & 0 \\ 0 & \kappa_{22}\end{array}\right]$.

In the isotropic case, $\kappa_{11}=\kappa_{22}=\kappa$. The anisotropic case considered later is $\kappa_{11} / 2=2 \kappa_{22}=\kappa$, which favors boundaries extended parallel to the habit plane. Recall from (C.16) that surface energy $\Gamma \propto \kappa^{1 / 2}$. The rationale for anisotropic surface energy is that twinning dislocations at a moving portion of the boundary (i.e., an incoherent interface in the terminology of Kosevich and Boiko [9]) would contribute core and elastic energies to the total surface energy of the interface. In contrast, the fully formed (i.e., coherent) twin boundary surface would have less energy than such a moving portion because it does not contain energy of dislocations, just stacking fault energy associated with reflection of the lattice across the twin boundary. The anisotropic case considered here will cause the twin to elongate in the direction of $\mathbf{s}$ and shorten in the direction of $\mathbf{m}$, in order to decrease the contribution of $\kappa_{11}\left(\nabla_{1} \eta\right)^{2}$ to the energy in (48).

The equilibrium thickness $l$ over which atoms deviate from their ideal positions is taken as $1 \mathrm{~nm}$, corresponding to about five $\{\overline{1} 012\}$ planes. This value follows from theoretical studies of perturbed atomic coordinates in twinned hexagonal close-packed structures [45]. The same characteristic thickness value $(1 \mathrm{~nm})$ has been used in phase field models of other materials [20]. In one set of calculations that follow, a sharper interface $(l=0.1 \mathrm{~nm})$ is also explored.

\subsection{Simulations and results}

Considered is a circular twin nucleus of initial radius $a_{0}=3 \mathrm{~nm}$, embedded in a much larger rectangular domain $\Omega$ of dimensions $L \times H$. Initially, a homogeneous displacement gradient field $\nabla \mathbf{u}$ is applied everywhere in $\Omega$. Particular boundary conditions are applied on $\partial \Omega$, as discussed in more detail below. The order 


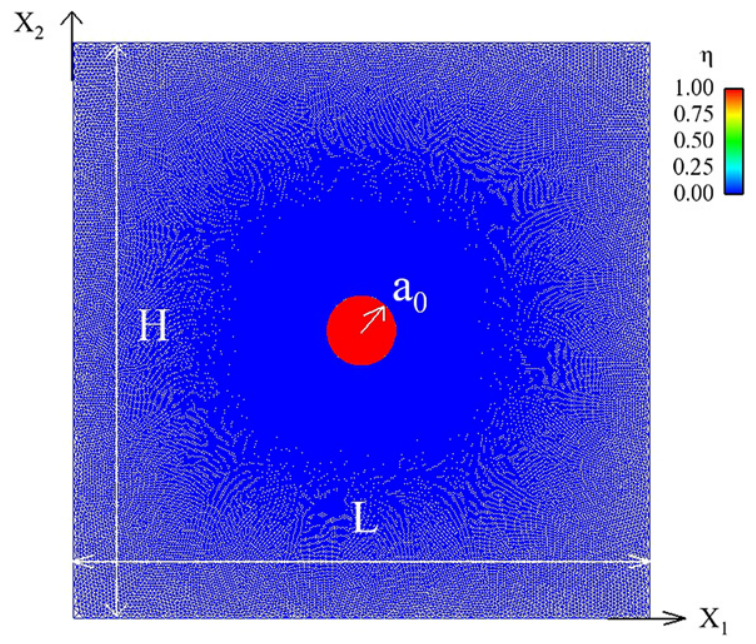

Fig. 2. Finite element mesh and initial conditions, undeformed shape.

parameter and local displacements are then relaxed according to the conjugate gradient algorithm discussed in Section 4, as a local stationary point (specifically, a local minimum) of the total free energy of the system is sought. If the magnitude of the applied displacement gradient is smaller than a critical value, the inclusion will shrink and may even disappear, the latter scenario suggesting that a purely homogeneous elastic response corresponds to a minimum energy state. If the magnitude of applied displacement is larger than a critical value, the inclusion will tend to grow until it reaches a final size limited by interaction with the external boundary of the domain. Growth does not necessarily imply that a configuration with an inclusion of finite size possesses less total energy than would the case of homogeneous elastic deformation; i.e., the configuration associated with an inclusion can be metastable and may constitute a relative minimum of the total energy.

Recall from Fig. 1 that $\theta$ denotes the orientation of the habit plane, i.e., the plane of twinning shear. Specifically, lattice orientation vectors are, in vector form,

$\mathbf{s}=[\cos \theta, \sin \theta]^{T}, \quad \mathbf{m}=[-\sin \theta, \cos \theta]^{T}$.

Recall also from (57) that the mechanical driving force for twinning is $\tau=\sigma:(\mathbf{s} \otimes \mathbf{m})$. From (69), it follows that for a pure shear stress state (e.g., $\sigma_{12}$ nonzero), $\tau=\sigma_{12}\left(\cos ^{2} \theta-\sin ^{2} \theta\right)$; for a pure tension stress state (e.g., $\sigma_{22}$ nonzero), $\tau=\sigma_{22} \sin \theta \cos \theta$.

Three kinds of boundary conditions are considered. The first is simple shear with Dirichlet conditions on the order parameter along $\partial \Omega$ :

$\left\{u_{1}=\gamma X_{2}, u_{2}=0, \eta=0\right\} \quad \forall X_{1}, X_{2} \in \partial \Omega$.

The second is shear with Neumann (free) conditions on traction and order parameter along the lateral edges of $\partial \Omega$ :

$u_{1}=\gamma X_{2}, \quad u_{2}=0 \quad \forall\left[X_{1},\left(X_{2}=0, H\right)\right]$,

$\mathbf{t}=\boldsymbol{\sigma} \mathbf{n}=\mathbf{0}, \quad \mathbf{n} \cdot \nabla \eta=0 \quad \forall\left[\left(X_{1}=0, L\right), X_{2}\right]$.

The third is simple tension with Neumann (free) conditions on traction and order parameter along the lateral edges of $\partial \Omega$ :

$u_{1}=0, \quad u_{2}=\varepsilon X_{2} \quad \forall\left[X_{1},\left(X_{2}=0, H\right)\right]$,

$\mathbf{t}=\boldsymbol{\sigma} \mathbf{n}=\mathbf{0}, \quad \mathbf{n} \cdot \nabla \eta=0 \quad \forall\left[\left(X_{1}=0, L\right), X_{2}\right]$.

In (70) and (71), $\gamma$ is the magnitude of applied shear, equal to twice the applied shear strain. In (72), $\epsilon$ is the applied stretch, equal to the applied tensile strain.

A representative finite element mesh is shown in Fig. 2. The mesh is highly refined towards the center where the inclusion is initially located. In simulations that follow, numerous elements resolve the thickness of the boundary of the inclusion in its equilibrium configuration, enabling accurate resolution of spatial gradients of the order parameter. The mesh shown in Fig. 2 includes 257,296 linear triangular elements. Some simulations were repeated with a finer mesh of 503,062 linear triangular elements; unless noted otherwise, results of interest are insensitive to mesh density.

The initial radius of the twin embryo (inclusion) is set at $a_{0}=$ $3 \mathrm{~nm}$ for the following reasons:

- According to the analytical sharp interface solution (Appendix E), a bifurcation from circular to elliptical shape occurs for a radius of $3.17 \mathrm{~nm}$, corresponding to the equality conditions in (E.9). Therefore, for ease of validation of the numerical technique with the analytical solution, circular inclusions with radii smaller than $3.17 \mathrm{~nm}$ must be considered. Comparison of larger nuclei would require consideration of elliptical shapes (i.e., aspect ratios $\omega<1$ ) and would unduly complicate comparisons with the analytical solution.

- According to atomic simulations of twin structures and twinning dislocations in $\mathrm{Mg}$, the minimum equilibrium size of a $\langle 1011\rangle\{\overline{1012}\}$ twin embryo is 17 atomic layers $(3.32 \mathrm{~nm})$ thick $[13,14]$. Shapes of twin embryos modeled using both DFT and empirical MD potentials $[13,14]$ were not always circular cylinders, but areas of twin embryos were reasonably close to those of a circle of radius $3 \mathrm{~nm}$. Unlike atomic simulations, the present phase field simulations do not resolve individual dislocation lines, but length scales involved are about the same.

- The equilibrium thickness $l$ of the twin boundary is $1 \mathrm{~nm}$. If $a_{0} \ll 3 \mathrm{~nm}$ were chosen, the boundary thickness would encroach on the center of the twin. If $a_{0} \gg 3 \mathrm{~nm}$ were chosen, computational cost associated with resolving the twin boundary would become prohibitive.

The analytical solution in (E.9) with inclusion radius $a_{1}=a_{2}=$ $\left(A_{T} / \pi\right)^{1 / 2}=3 \mathrm{~nm}$ corresponds to a critical stress of $\tau_{\infty} / \mu=$ 0.038 . This solution has been derived for an inclusion embedded in an infinite domain, with a sharp interface between matrix and inclusion. Thus, if effects of finite domain size and a diffuse interface are not strong, in the present phase field simulations applied shear $\gamma \gg 0.038$ should result in growth of the twin, while $\gamma \ll 0.038$ should result in decay [for cases in which simple shear boundary conditions (70) are applied]. For shear loading, the applied stress is defined as the following volume average, using the divergence theorem and the first of (52):

$\tau_{A}=\frac{1}{\Omega} \int_{\partial \Omega} t_{1} X_{2} \mathrm{~d} S=\frac{1}{\Omega} \int_{\Omega} \sigma_{12} \mathrm{~d} \Omega$.

Shown in Fig. 3 are simulation results for applied shear (a) $\gamma=0.01$ and (b) $\gamma=0.10$. In each case, $\theta=0$ and boundary conditions (70) apply. Notice that decay [Fig. 3(a), $\gamma=0.01 \ll$ 0.038 ] to a homogeneous medium (no twin) and growth [Fig. 3(b), $\gamma=0.10 \gg 0.038]$ occur in agreement with the analytical solution. For $\gamma=0.01$, the twin nucleus shrinks and disappears; for $\gamma=0.10$, the inclusion grows until it is repelled by rigid outer boundaries $\partial \Omega$ where $\eta=0$ is prescribed.

Next sought are critical applied strains for twin nucleation for the cases listed in Table 2, which encompass various boundary conditions, material properties, and lattice orientations. Critical strains are obtained in practice by seeding the domain with an inclusion of initial radius $a_{0}=3 \mathrm{~nm}$, and then applying the deformation [ $\gamma$ for shear, $\epsilon$ for tension as in (70)-(72)] in small increments of magnitude 0.001 . For each increment, system degrees of freedom relax in conjunction with energy minimization, performed numerically via the conjugate gradient technique. It was found that in the first few hundred conjugate gradient 


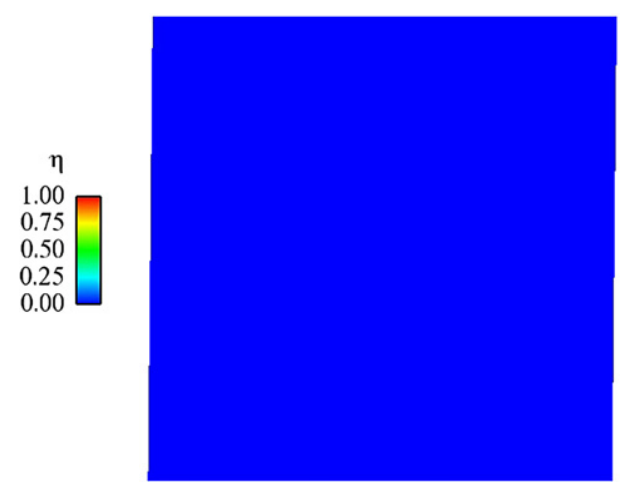

(a) $\gamma=0.01$.

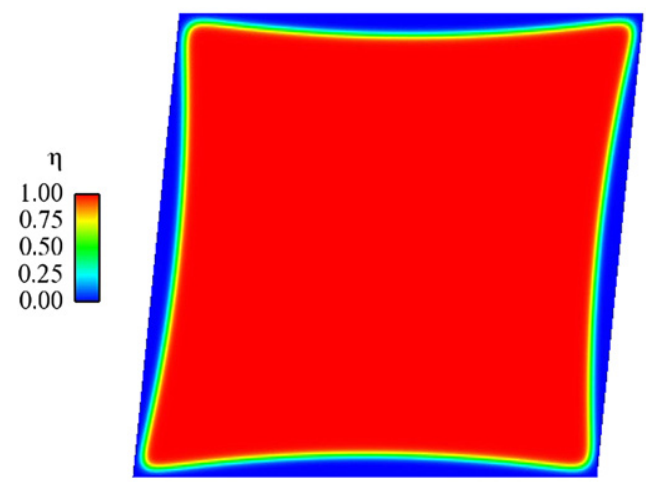

(c) $\gamma=0.10$.

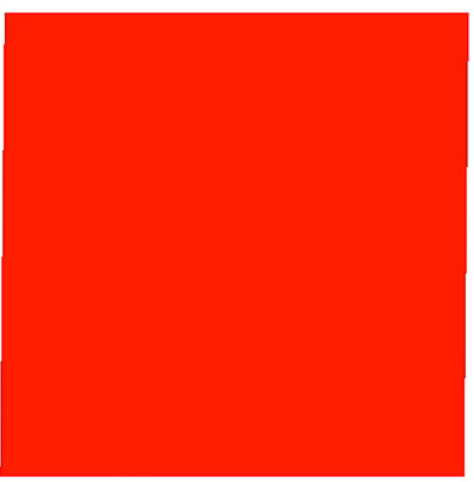

(b) $\gamma=0.01$
Stress $[\mathrm{GPa}]$

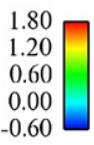

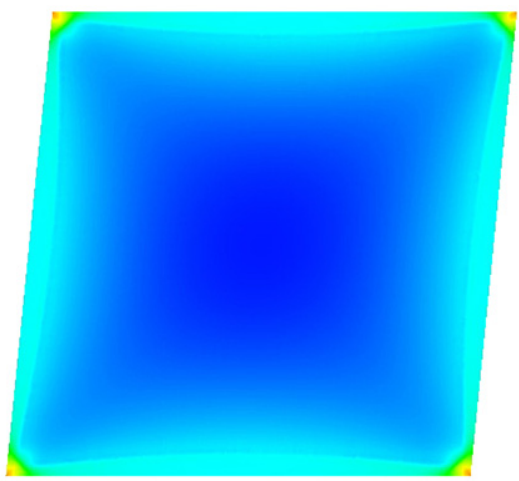

(d) $\gamma=0.10$

Fig. 3. Order parameter $\eta$ and shear stress $\sigma_{12}$ : (a), (b) $\gamma=0.01$ (twin disappears) and (c), (d) $\gamma=0.10$ (twin grows).

Table 2

Phase field simulations and predicted critical strain and stress for twin nucleation. For Case $4, \kappa_{11} / 2=2 \kappa_{2}=\kappa$. For Case $6, \gamma_{C}=\gamma / 2$ and $\tau_{A}$ is divided by 2 . For Case 7, $\gamma_{C}=\varepsilon$ and $\tau_{A}=\sigma / 2$, with $\sigma$ the average normal stress.

\begin{tabular}{lllllllll}
\hline Case & $\begin{array}{l}\theta \\
(\mathrm{rad})\end{array}$ & $\begin{array}{l}l \\
(\mathrm{~nm})\end{array}$ & $\kappa_{11} / \kappa_{22}$ & $L / H$ & Boundary & Loading & $\gamma_{C}$ & $\tau_{A} / \mu$ \\
\hline 1 & 0 & 1 & 1 & 1 & Eq. (70) & shear & 0.050 & 0.020 \\
2 & 0 & 1 & 1 & 1 & Eq. (71) & shear & 0.050 & 0.000 \\
3 & 0 & 1 & 1 & 1.5 & Eq. (70) & shear & 0.049 & 0.012 \\
4 & 0 & 1 & 4 & 1 & Eq. (70) & shear & 0.046 & 0.023 \\
5 & 0 & 0.1 & 1 & 1 & Eq. (70) & shear & 0.049 & 0.044 \\
6 & $\pi / 6$ & 1 & 1 & 1 & Eq. (70) & shear & 0.050 & 0.042 \\
7 & $\pi / 4$ & 1 & 1 & 1 & Eq. (72) & tension & 0.045 & 0.005 \\
\hline
\end{tabular}

iterations, substantial changes in size and shape of the twin occur in response to the prescription of an initially sharp interface, as the interface diffuses to a width commensurate with parameter $l$ of the phase field model. In subsequent energy minimization iterations, the twin embryo either grows or decays, with magnitude of the rate of growth or decay versus number of iterations increasing with increasing strain difference from the critical strain. The minimum applied strain for which monotonic decay (to null size) does not occur is deemed the critical strain $\gamma_{C}$. The accuracy of $\gamma_{C}$ obtained via this method is limited to the applied strain increment magnitude of 0.001 . Qualitative profiles of total energy versus inclusion (twin) size for two values of applied shear are shown in Fig. 4. At a given shear $\gamma$, when $a<a_{C}$ the twin will tend to shrink and disappear, following the energy surface downhill to the left. When $a>a_{C}$ the twin will tend to grow until impeded by external boundaries, following the energy surface downhill to the right. For each of the simulation cases considered in Table 2, application of shear $\gamma=\gamma_{c}-0.001$ resulted in shrinkage and disappearance of the initial twin nucleus of radius $3 \mathrm{~nm}$.

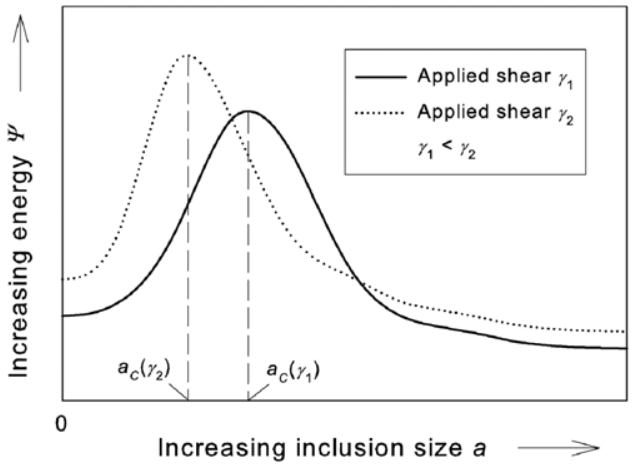

Fig. 4. Sketch of system energy versus inclusion size for two values of applied shear.

Critical strains $\gamma_{C}$ and corresponding average shear stresses $\tau_{A}$ are listed in Table 2. For cases 1-5, critical stresses are defined as volume averages $\tau_{A}$ in (73) at the applied strain corresponding to growth. For case 6 (shear, $\theta=\pi / 6$ ), results in Table 2 are normalized via multiplication by the factor $\left(\cos ^{2} \theta-\sin ^{2} \theta\right)=1 / 2$ to enable comparison with cases 1-5. For case 7 (tension, $\theta=$ $\pi / 4)$, critical strain and stress are defined respectively as $\gamma_{C}=\varepsilon$ and $\tau_{A}=(\sin \theta \cos \theta) \int_{\Omega} \sigma_{22} \mathrm{~d} \Omega=\sigma / 2$.

Fig. 5 shows equilibrium order parameter contours for each simulation at the applied critical strain. Fig. 6 shows corresponding shear stresses. For cases 1-6, the local shear stress shown is $\sigma_{12}$; for case 7 , the stress shown is that acting on a $45^{\circ}$ plane, $\left(\sigma_{22}-\sigma_{11}\right) / 2$. The following observations from Table 2 and Figs. 5 and 6 are noteworthy:

Case 1 . The equilibrium shape of the twin embryo is non-circular and slightly concave as a result of the applied deformation and boundary conditions. The twin grows horizontally until repelled 


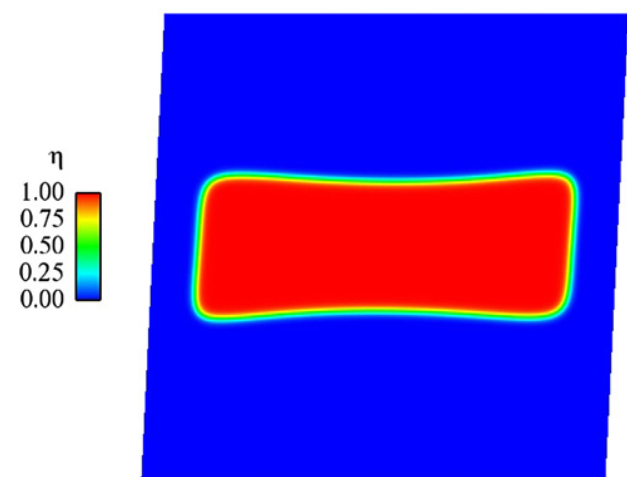

(a) Case 1 .

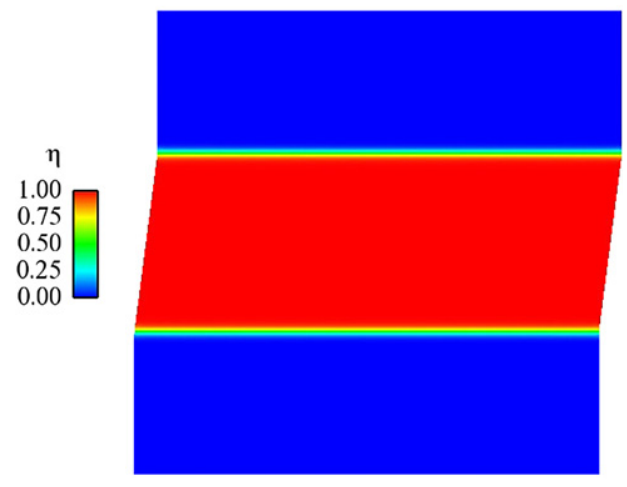

(b) Case 2
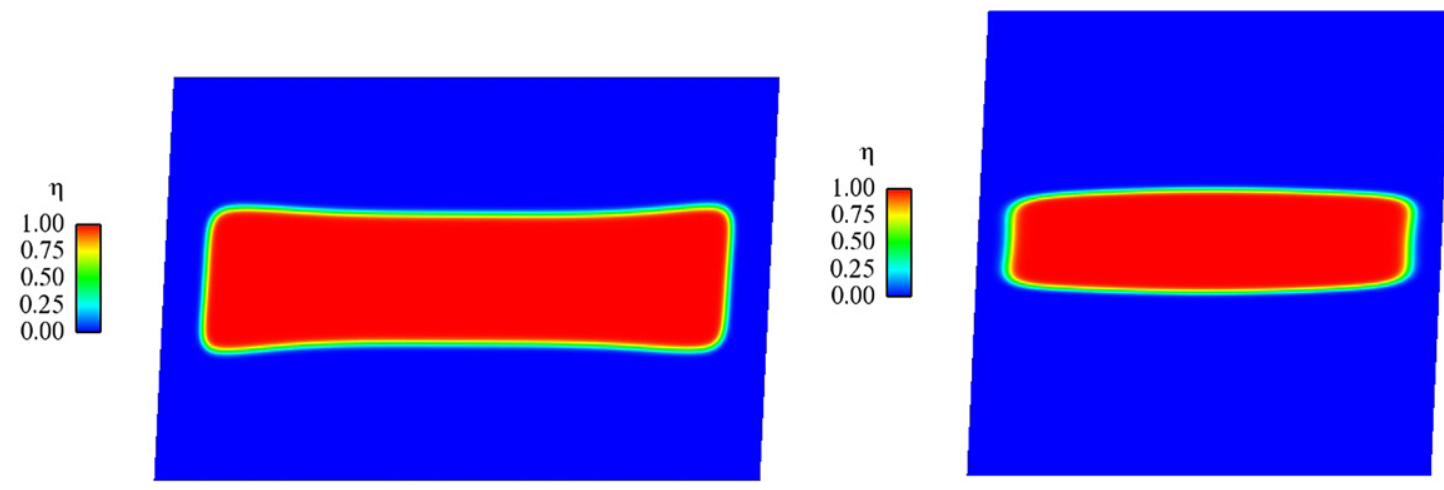

(c) Case 3.

(d) Case 4 .
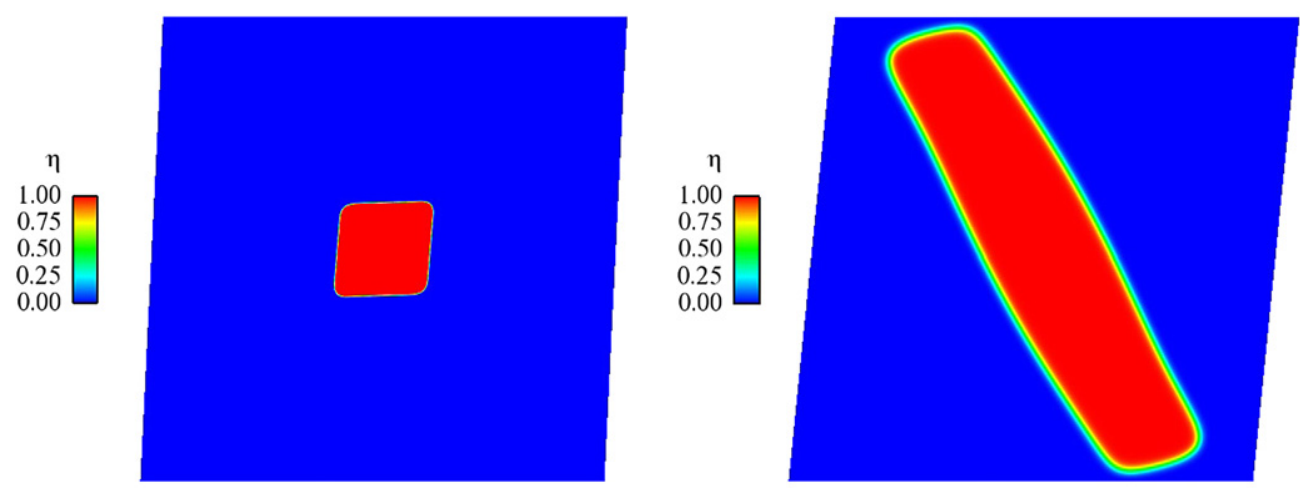

(e) Case 5 .

(f) Case 6.

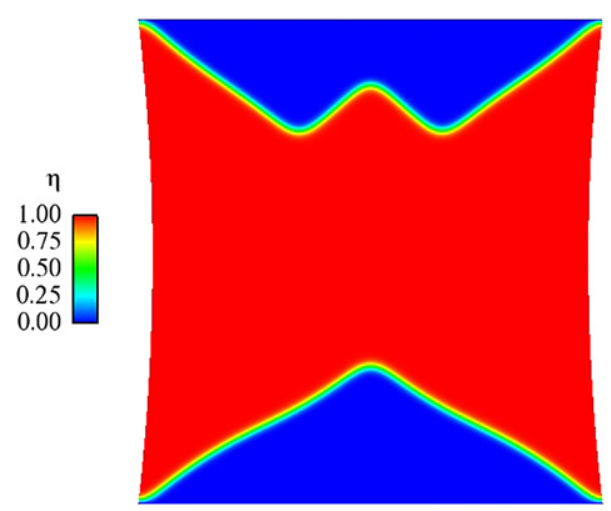

(g) Case 7

Fig. 5. Order parameter $\eta$ at applied critical strains corresponding to Table 2: (a) Case 1, (b) Case 2, (c) Case 3, (d) Case 4, (e) Case 5, (f) Case 6 and (g) Case 7. 


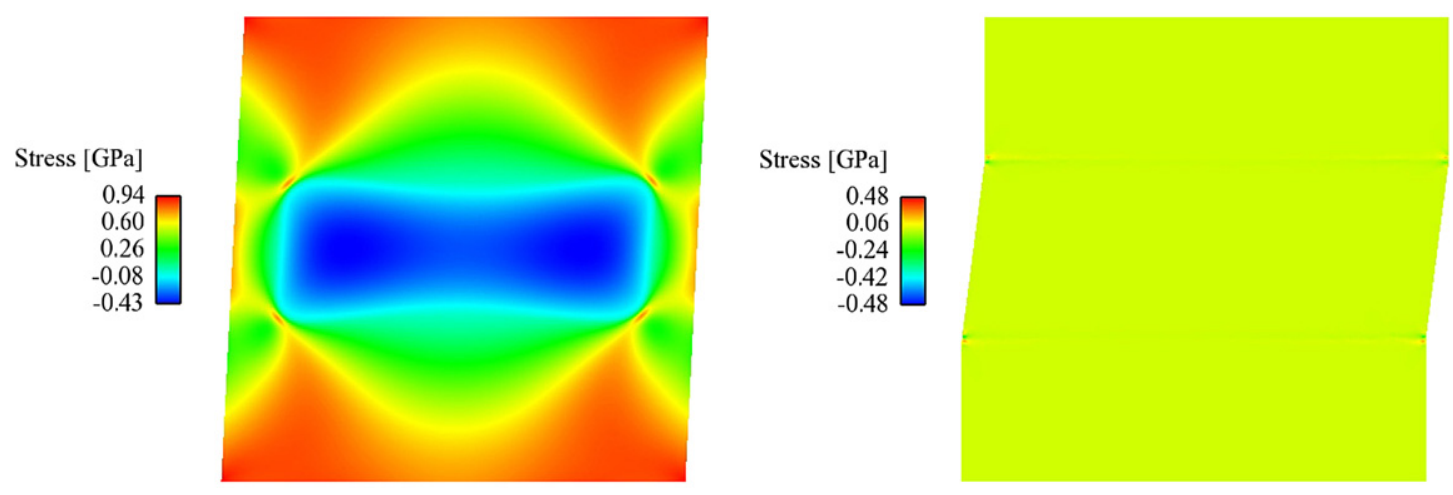

(a) Case 1.

(b) Case 2 .

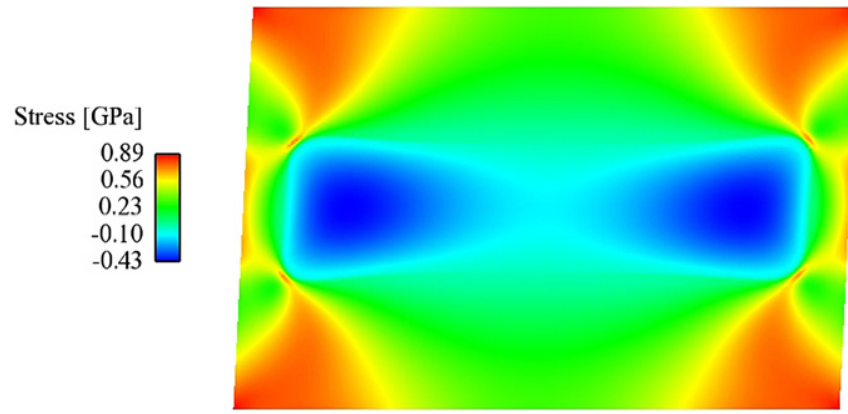

(c) Case 3.

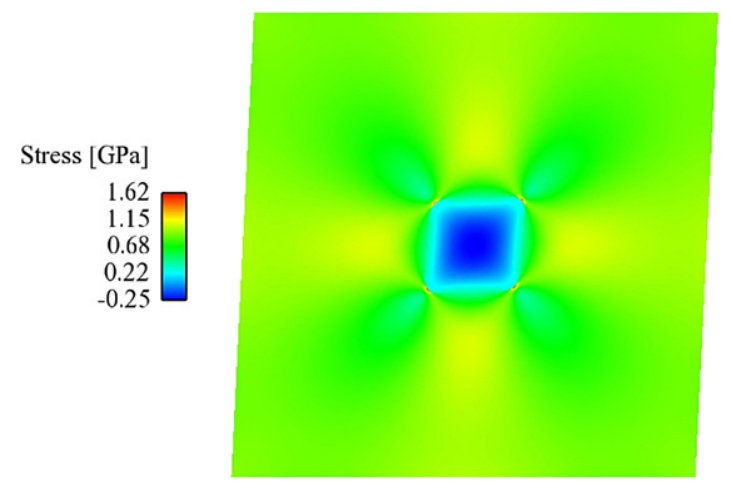

(e) Case 5 .
Stress $[\mathrm{GPa}]$

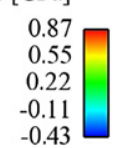

(d) Case 4 .
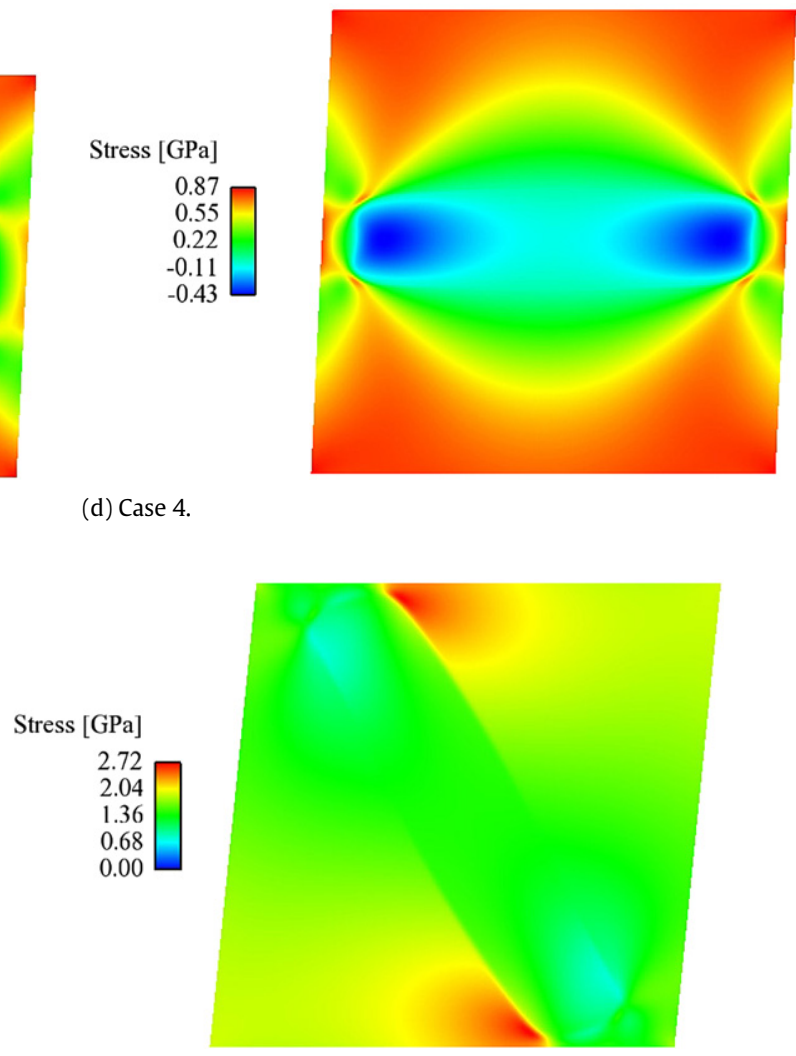

(f) Case 6 .

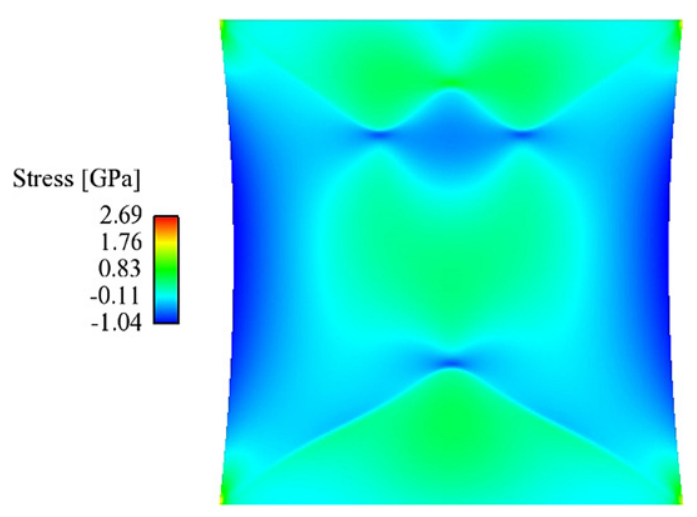

(g) Case 7.

Fig. 6. Shear stress at applied critical strains corresponding to Table 2: (a) Case 1, (b) Case 2, (c) Case 3, (d) Case 4, (e) Case 5, (f) Case 6 and (g) Case 7. 


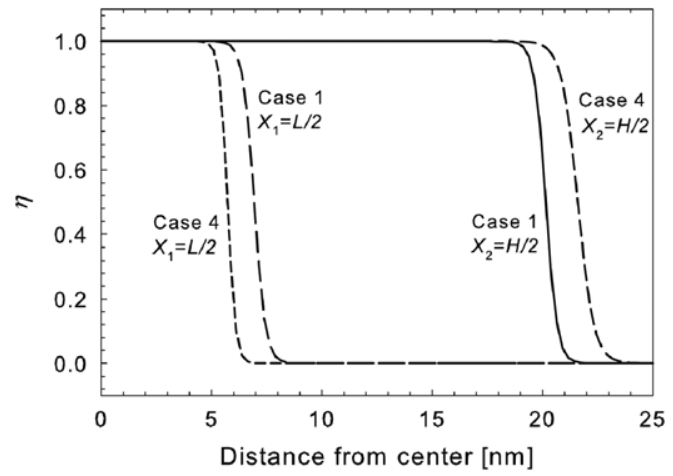

Fig. 7. Profiles of order parameter along horizontal mid-plane $X_{2}=H / 2$ and vertical mid-plane $X_{1}=L / 2$ for Case 1 (isotropic surface energy) and Case 4 (anisotropic surface energy).

by the lateral boundaries at $X_{1}=0, L$. The critical shear displacement gradient $\gamma_{c}=0.05$. The applied average shear stress is significantly smaller than the stress that would be obtained for a homogeneous elastic slab with no inclusion, i.e., $\tau_{A} /\left(\mu \gamma_{C}\right) \approx 0.4<$ 1. Fig. 7 shows the value of the order parameter along reference coordinates measured from the center of the inclusion: $X_{2}=H / 2$ (the horizontal mid-plane) and $X_{1}=L / 2$ (the vertical mid-plane). From Fig. 7, along either mid-plane, the thickness of the region over which $0.1 \lesssim \eta \lesssim 0.9$ is very close to parameter $l=1 \mathrm{~nm}$.

Case 2. Here the lateral boundaries are assigned free Neumann conditions. The twin grows horizontally until it fully spans the domain. The applied critical strain $\gamma_{C}=0.05$ is identical to that of Case 1 , but the average stress is negligible: $\tau_{A} /\left(\mu \gamma_{C}\right) \approx 0$. The transformation strain and rotation of twin fully accommodate the imposed shear deformation, and local shear stresses vanish except at a few locations near twin boundaries, as is clear from Fig. 6(b).

Case 3. The equilibrium shape of the twin embryo is longer in the horizontal $\left(X_{1}\right)$ direction, but otherwise is similar to that of Case 1 . The applied critical strain $\gamma_{C}=0.049$ is slightly smaller than that of Case 1 (see Table 2 ), and the ratio of average stress to critical strain is $\tau_{A} /\left(\mu \gamma_{C}\right) \approx 0.24$. Increasing the aspect ratio $L / H$ of the domain from 1 to 1.5 apparently has a small effect on the critical strain for homogeneous nucleation.

Case 4 . The equilibrium shape of the twin embryo is wider (in the $X_{1}$-direction) and flatter (in the $X_{2}$-direction) than that of Case 1 , as is clear from profiles of the order parameter in Fig. 7. The thickness of the twin boundary is larger along the left and right (shorter) sides and smaller along the top and bottom (longer) sides, as also quantified in Fig. 7. In contrast to the isotropic cases (e.g., Case 1), prescription of anisotropic surface energy $\left(\kappa_{11} / 2=2 \kappa_{22}=\kappa\right)$ results in a convex rather than concave shape. The applied critical strain $\gamma_{C}=0.046$ is smaller than that of Case 1 , and the ratio of average stress to critical strain is larger: $\tau_{A} /\left(\mu \gamma_{C}\right) \approx 0.5$.

Case 5. The equilibrium shape of the twin embryo is that of a parallelogram, confined to the center of the domain where the mesh is sufficiently refined to resolve the twin boundary interface. The thickness of the interface is much smaller than that of Case 1, in agreement with parameter choice $l=0.1 \mathrm{~nm}$. The applied critical strain $\gamma_{C}=0.049$ is very close to that of Case 1 , and the average shear stress $\tau_{A} /\left(\mu \gamma_{C}\right) \approx 0.9$. Shear stress concentrations of $1.5 \mathrm{GPa}$ arise at four corner locations along the twin-matrix interface. For this case only, the equilibrium shape is mesh sensitive. The critical strain is not deemed mesh sensitive, however, since applied strains $\gamma_{C}<0.049$ resulted in decay and disappearance of the initial twin embryo.

Case 6. The equilibrium shape of the twin embryo differs from that in Case 1; here, the twin appears rotated such that one axis,

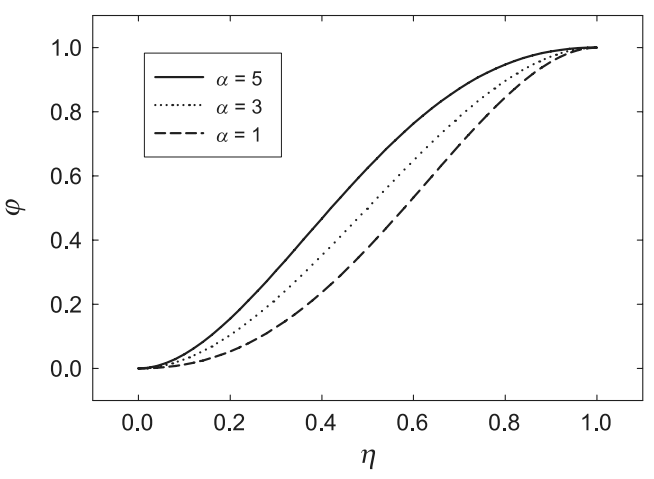

Fig. 8. Interpolation function of (9) [35].

in reference coordinates, is aligned normal to the direction $\mathbf{s}$ of twinning shear $\left(30^{\circ}\right.$ from horizontal). The applied critical strain $\gamma_{C}=\gamma / 2=0.050$ is identical to that of Case 1 , and $\tau_{A} /\left(\mu \gamma_{C}\right) \approx$ 0.84. The applied shear stress is larger in Case 6 than in Case 1 because the applied shear $\gamma$ here is 0.10 rather than 0.05 .

Case 7 . The equilibrium shape of the twin embryo differs drastically from that in Case 1 . The twinned region is large and nearly symmetric about vertical mid-plane $X_{1}=L / 2$. The applied critical tensile strain $\gamma_{C}=\varepsilon=0.045$, the average applied stress $\tau_{A}=$ 0.005 , and the ratio of average stress to critical strain $\tau_{A} /\left(\mu \gamma_{C}\right) \approx$ 0.11 .

In results discussed thus far, interpolation function $\varphi$ of (9) [35] is prescribed as $\alpha=3$. Exploratory simulations were also conducted with $\alpha=1$ and $\alpha=5$; corresponding interpolation functions are plotted in Fig. 8. Results of interest are not substantially different with different choices of $\alpha$. For example, as shown in Fig. 9(a), profiles of the order parameter versus distance from the inclusion along mid-planes $X_{1}=L / 2$ and $X_{2}=H / 2$ do not depend strongly on $\alpha$; profiles shift only slightly to the left (i.e., towards the inclusion center) with increasing $\alpha$. The middle value explored, $\alpha=3$, for which (9) degenerates to a cubic polynomial, provides the anti-symmetry conditions $\varphi(1-\eta)=$ $1-\varphi(\eta)$ and would seem most physically appropriate for modeling flat interfaces. For $\alpha=1,3$, or 5 , profiles of transformation shear $\varphi \gamma_{0}$ are indistinguishable in Fig. 9(b).

\subsection{Discussion}

Results in Section 5.3, Table 2, and Figs. 5 and 6 suggest the following trends regarding nucleation of a twin embryo of minimum initial radius $3 \mathrm{~nm}$ :

- The critical far-field (i.e., applied) shear displacement gradient for nucleation and growth is $0.045 \leq \gamma_{C} \leq 0.050$, and appears fairly insensitive to type of boundary condition (i.e., Dirichlet or Neumann in (70) or (71)), aspect ratio of the domain $\Omega$, surface energy anisotropy $\kappa_{11} / \kappa_{22}$, twin boundary thickness $l$, and initial lattice orientation $\theta$.

- For shear loading conditions perfectly aligned with the geometry of the twin system $(\theta=0)$, the average shear stress after nucleation and growth is $0<\tau_{A} / \mu \leq 0.044$, is smaller than that corresponding to what would be observed for homogeneous elastic shear (i.e., for no twin), and can be sensitive to type of boundary condition.

- Equilibrium and near-equilibrium shapes of twins are noncircular and are sensitive to surface energy anisotropy ratio $\kappa_{11} / \kappa_{22}$, twin boundary thickness parameter $l$, boundary conditions, and loading mode (e.g., shear or tension).

Table 3 compares twin nucleation criteria resulting from the present simulations with those of other studies. The lower bound 
a
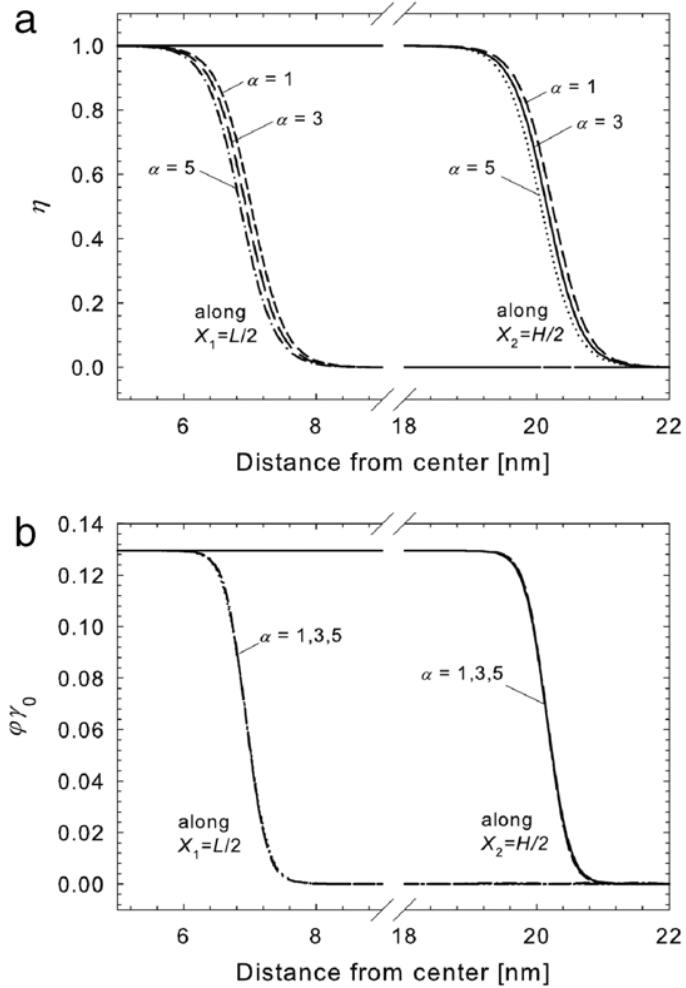

Fig. 9. Profiles of (a) order parameter $\eta$ and (b) twinning shear $\varphi \gamma_{0}$ along vertical $\left(X_{1}=L / 2\right)$ and horizontal $\left(X_{2}=H / 2\right)$ mid-planes for Case $1(\gamma=0.05)$ for different values of interpolation parameter $\alpha$.

Table 3

Critical shear deformation for twin nucleation or growth: comparison with other models.

\begin{tabular}{llll}
\hline Model & Reference & Remarks & $\gamma_{C}$ \\
\hline $\begin{array}{l}\text { Phase field } \\
\text { Elastic inclusion }\end{array}$ & This work & $\begin{array}{l}\text { Simulations 1-7 [ Table 2] } \\
\text { Analytical solution, }\end{array}$ & $0.045-0.050$ \\
& {$[30]$} & $\begin{array}{l}\text { infinite medium } \\
\text { inge }\end{array}$ & 0.038 \\
$\begin{array}{l}\text { Theoretical stress } \\
\text { Atomistic } \\
\text { calculation }\end{array}$ & {$[25,48]$} & $\begin{array}{l}\gamma_{C}=\gamma_{0} /(2 \pi) \\
\text { Glide of } \\
1 / 17[10 \overline{1} 1](\overline{1012})\end{array}$ & 0.021 \\
& & $\begin{array}{l}1 / 24] \\
\text { partial dislocation }\end{array}$ & 0.004 \\
\hline
\end{tabular}

on the present results is in fair agreement with the analytical model of Lee and Yoo [30] outlined in Appendix E, where here the notation $\gamma_{c}=\tau_{\infty} / \mu$. Differences between phase field results and the analytical elasticity solution of Appendix $E$ are apparently due to resolution of finite boundary conditions and finite interfacial thickness in the former. The lower bound on critical shear strain obtained through the phase field approach exceeds that from "theoretical strength" $\gamma_{0} /(2 \pi)$ associated with motion of twinning partial dislocations $[25,48]$, and far exceeds the stress from empirical lattice statics calculations [23,24]. The latter lattice statics approaches consider motion of a single partial dislocation in a long planar twin boundary interface and do not account for the finite size and curved shape of the twin nucleus. This may account for the discrepancies in values between atomic simulation results and the first two models listed in Table 3.

Further qualitative agreement between the present model and analytical elasticity solutions is apparent upon consideration of simulation results in Fig. 3 and the sketch in Fig. 4. Recall that $\gamma_{C}$ corresponds to an unstable equilibrium point, i.e., a saddle point on the energy surface parameterized in terms of both size and shape of the inclusion. At the saddle point, the total energy is minimized with respect to shape, but maximized with respect to size $[31,32]$. In agreement with the analytical solution of Appendix E, applied strains less than (or greater than) the critical strain result in unstable decay (or growth) of the twin embryo. The saddle point configuration is not attained in the conjugate gradient calculations (witness complete disappearance or substantial growth of inclusions in Fig. 3) because the algorithm seeks energy minima rather than saddle points. Recall that the total free energy is a non-convex functional of the order parameter, as is obvious from the contribution of the double-well potential function in (20). It is expected that (28) may exhibit one, many, or no stationary points (i.e., minima, maxima, and/or saddle points), as is characteristic of phase change problems involving non-convex free energy functionals [20,31]. Examination of the rate of change of total free energy versus iterations for each simulation indicate that, after a sufficient number of conjugate gradient iterations, the state thus attained corresponds to a local, and possibly global free, energy minimum. The same end result could presumably, but not necessarily, be obtained via use of a kinetic approach to evolve the order parameter such as (D.3) of Appendix D, wherein the right side of (D.3) vanishes at every material point in the domain in the equilibrium state when a stationary condition on the total free energy has been attained.

Remarks regarding assumptions and limitations of the present phase field approach are in order. The general theory developed in Section 2 accounts for geometric nonlinearity (i.e., large deformations) and anisotropy. Simulations presented in Section 5 incorporate geometric linearity (Section 3) and isotropic elastic constants. Comparison of (39) with (57) shows that, when elastic strains are small, the difference in driving force for twinning $\tau$ between geometrically nonlinear and linear theories is a factor of $\left(\mathbf{F}^{\eta}\right)^{-1}$, which in turn is on the order of twinning shear $\gamma_{0}, 0.1295$ for the twin system under consideration in $\mathrm{Mg}$ (Table 1). The difference between the two theories would be more severe in cubic crystals, where for the usual twinning mode, $\gamma_{0}=2^{-1 / 2}$. As remarked in Section 5.1, anisotropy of elastic constants in $\mathrm{Mg}$ is low [47]. Furthermore, atomic simulations have demonstrated qualitative and reasonably quantitative agreement for many features of twins in hexagonal crystals modeled using pair potentials [23] which cannot correctly describe the five independent anisotropic elastic constants, and using many-body potentials [24] which can correctly describe anisotropic elastic constants. The present phase field method does not enable resolution of atomic details of defect structures afforded by quantum or molecular mechanics models. However, a major advantage of the present approach is that very few material parameters are needed: at minimum, two elastic constants and two other constants related to twin boundary energy and twin boundary thickness. Furthermore, the same continuum theory can be used to address systems of much larger size than can be treated using discrete atomic techniques, so long as the numerical grid is sufficient to resolve gradients of the order parameter at interfaces. The present work has considered only a 2D idealization of twinning in Mg. Real deformation processes can involve three spatial dimensions and multiple twin systems, up to six $\langle 10 \overline{1} 1\rangle\{1012\}$ systems in Mg [34]. Future work may consider 3D simulations and multiple twins, following the theory in Appendix B.

\section{Conclusions}

A nonlinear theory has been developed to address mechanical twinning. The general theory accounts for large deformations with a focus on equilibrium thermodynamics. A geometrically linear version of the theory has been implemented in finite element simulations of homogeneous twin nucleation. The application appears to be the first documented phase field study of homogeneous 
twin nucleation in Mg single crystals. Results are in fair agreement with analytical solutions for critical applied strains associated with nucleation and subsequent unstable growth of a small twin embryo. Critical shear displacement gradients for nucleation and growth of a cylindrical twin of size $3 \mathrm{~nm}$ are predicted on the order of $0.045-0.050$. Numerical simulations enable consideration of effects of boundary conditions, finite domain sizes, and surface energy anisotropy not amenable to analytical solutions. Results demonstrate that minimum applied stresses necessary for twin nucleation and growth can be sensitive to boundary conditions associated with mechanical and order parameter fields, and that equilibrium shapes of twin nuclei can be sensitive to surface energy anisotropy.

\section{Appendix A. Existence of minimizers of energy functional (10)}

Existence of minimizers of energy functionals (10) can be demonstrated under conditions of convexity, quasiconvexity or polyconvexity of the underlying energy density functions. The present treatment is limited to convex or polyconvex energy density functions, important classes of functions for applications. Provided here is a brief summary of essential results; textbooks on the calculus of variations (e.g., [39]) should be consulted for a more comprehensive treatment.

\section{A.1. Convex energy density functions}

Let $W(\mathbf{X}, \mathbf{F}, \eta)$ and $f(\mathbf{X}, \eta, \nabla \eta)$ be convex in $\mathbf{F}$ and $\nabla \eta$, respectively. In addition, let $g(\mathbf{X}, \eta, \mathbf{F}, \nabla \eta)=W(\mathbf{X}, \mathbf{F}, \eta)+$ $f(\mathbf{X}, \eta, \nabla \eta)$. It is easily verified that $g$ is a convex function of $(\mathbf{F}, \nabla \eta)$. Consequently, it can be shown $[38,39]$ that problem (10) possesses a solution provided function $g$ satisfies the following coercivity condition:

$g(\mathbf{X}, \eta, \mathbf{F}, \nabla \eta) \geq \alpha(\mathbf{X})+\beta\left(|\mathbf{F}|^{2}+|\nabla \eta|^{2}\right)^{p / 2}$

for almost every $\mathbf{X} \in \Omega$, for every $\mathbf{F} \in \mathbb{R}_{+}^{3 \times 3}, \beta>0$ and $\nabla \eta \in \mathbb{R}^{3}$ and for some absolutely integrable function $\alpha$ and $p>1$. $|\bullet|$ denotes the two-norm.

\section{A.2. Polyconvex energy density functions}

A function $g: \mathbb{R}^{m \times n} \rightarrow \overline{\mathbb{R}}=\mathbb{R} \cup\{+\infty\}$ is said to be polyconvex if there exists a convex function $h: \mathbb{R}^{\tau(n, m)} \rightarrow \overline{\mathbb{R}}$, such that

$g(\mathbf{A})=h[T(\mathbf{A})]$

where $T: \mathbb{R}^{m \times n} \rightarrow \mathbb{R}^{\tau(n, m)}$ is defined as

$T(A)=\left(\mathbf{A}, \operatorname{adj}_{2} \mathbf{A}, \ldots, \operatorname{adj}_{n \wedge m} \mathbf{A}\right)$.

Here, $n \wedge m=\min \{n, m\}$, $\operatorname{adj}_{s} \mathbf{A}$ denotes all $s \times s$ minors of $\mathbf{A} \in \mathbb{R}^{m \times n}, 2 \leq s \leq n \wedge m$, and

$\tau(n, m)=\sum_{s=1}^{n \wedge m} \frac{m ! n !}{(s !)^{2}(m-s) !(n-s) !}$.

In the context of conventional strain energy density functions $W: \mathbb{R}_{+}^{3 \times 3} \rightarrow \mathbb{R}$, the assumption of polyconvexity yields the wellknown functional form $W(\mathbf{F})=\tilde{W}\left(\mathbf{F}, \operatorname{cof} \mathbf{F}^{T}\right.$, det $\left.\mathbf{F}\right)$, where cof $\mathbf{A}$ denotes the cofactor of matrix $\mathbf{A}[39,40]$, and where $\tilde{W}$ is a convex function of its arguments.

Define $\mathbf{u}=(\eta, \chi)$ and its gradient

$[\nabla \mathbf{u}]=\left[\begin{array}{c}\nabla \eta \\ \mathbf{F}\end{array}\right]=\left[\begin{array}{lll}\nabla_{1} \eta & \nabla_{2} \eta & \nabla_{3} \eta \\ F_{11} & F_{12} & F_{13} \\ F_{21} & F_{22} & F_{23} \\ F_{31} & F_{32} & F_{33}\end{array}\right]$.
Let $g: \Omega \times \mathbb{R}^{4} \times \mathbb{R}^{4 \times 3} \rightarrow \bar{R}$ be defined

$g[\mathbf{X}, \mathbf{u}, \nabla \mathbf{u}]=g[\mathbf{X},(\eta, \chi),(\nabla \eta, \mathbf{F})]$.

Assume that $g$ in (A.6) is polyconvex in $\nabla \mathbf{u}$. Application of definitions (A.2)-(A.4), with $n=3, m=4, n \wedge m=3$ and $\tau(n, m)=34$, yields the representation $g(\mathbf{X}, \mathbf{u}, \nabla \mathbf{u})=$ $\tilde{g}\left(\mathbf{X}, \mathbf{u}, \nabla \mathbf{u}, \operatorname{adj}_{2} \nabla \mathbf{u}, \operatorname{adj}_{3} \nabla \mathbf{u}\right)$. Array $\operatorname{adj}_{2} \nabla \mathbf{u}$ contains components of cof $\nabla \mathbf{u}^{T}$ and determinants of all $2 \times 2$ sub-matrices of $\nabla \mathbf{u}$ of type

$\left[\begin{array}{ll}\nabla_{1} \eta & \nabla_{2} \eta \\ F_{11} & F_{12}\end{array}\right]$.

Similarly, array $\operatorname{adj}_{3} \nabla \mathbf{u}$ contains det $\mathbf{F}$ and determinants of the three $3 \times 3$ sub-matrices of $\nabla \mathbf{u}$ of type

$\left[\begin{array}{lll}\nabla_{1} \eta & \nabla_{2} \eta & \nabla_{3} \eta \\ F_{11} & F_{12} & F_{13} \\ F_{21} & F_{22} & F_{23}\end{array}\right]$

It is emphasized that $\tilde{g}$ is convex in its last three arguments for all $\mathbf{X} \in \Omega$ and $\mathbf{u} \in \mathbb{R}^{4}$ by the polyconvexity of $g$.

Notions of polyconvexity become further simplified if an additive decomposition of $g$ is used. Take $W$ polyconvex in $\mathbf{F}$, $W(\mathbf{X}, \mathbf{F}, \eta)=\tilde{W}\left(\mathbf{X}, \mathbf{F}, \operatorname{cof} \mathbf{F}^{T}\right.$, det $\left.\mathbf{F}, \eta\right)$, and $f$ convex in $\nabla \eta$; function $g$ is then represented as

$$
\begin{aligned}
g(\mathbf{X}, \mathbf{u}, \nabla \mathbf{u}) & =\tilde{g}\left(\mathbf{X}, \eta, \mathbf{F}, \operatorname{cof} \mathbf{F}^{T}, \operatorname{det} \mathbf{F}, \nabla \eta\right) \\
& =\tilde{W}\left(\mathbf{X}, \mathbf{F}, \operatorname{cof} \mathbf{F}^{T}, \operatorname{det} \mathbf{F}, \eta\right)+f(\mathbf{X}, \eta, \nabla \eta),
\end{aligned}
$$

and clearly satisfies the more general polyconvex form above. Furthermore, since $\tilde{W}$ and $f$ are both convex functions of their appropriate arguments, $\tilde{g}$ is a convex function of $\mathbf{F}, \operatorname{cof} \mathbf{F}^{T}$, det $\mathbf{F}$, and $\nabla \eta$, which, in turn, renders $g$ polyconvex.

The existence of minimizers of (10) with $g$ in (A.9) is assured provided the following coercivity condition is satisfied [39]:

$g(\mathbf{X}, \mathbf{u}, \nabla \mathbf{u}) \geq \alpha(\mathbf{X})+\sum_{s=1}^{3} \beta_{s}\left|\operatorname{adj}_{s} \nabla \mathbf{u}\right|^{p_{s}}$,

where $\alpha$ is an absolutely integrable function over $\Omega, \beta_{s}>0$, $p_{1} \geq 2, p_{2} \geq \frac{p_{1}}{p_{1}-1}$ and $p_{3}>1$. This condition can be expressed more explicitly as

$$
\begin{aligned}
g(\mathbf{X}, \mathbf{u}, \nabla \mathbf{u}) \geq & \alpha(\mathbf{X})+\beta_{1}\left(|\mathbf{F}|^{2}+|\nabla \eta|^{2}\right)^{p_{1} / 2} \\
& +\beta_{2}|\operatorname{cof} \mathbf{F}|^{p_{2}}+\beta_{3}(\operatorname{det} \mathbf{F})^{p_{3}}
\end{aligned}
$$

\section{Appendix B. Multiple twin systems}

The theory of Section 2 is extended to account for multiple twin systems, i.e., more than two phases. Denote scalar order parameter fields by $\eta_{i}(\mathbf{X}, t)$. Let $i=0,1,2, \ldots, n$, with $n+1$ the total number of phases. For a crystal with multiple twin systems, $n$ is the number of twin systems, $i=0$ is associated with the parent, and $i=1,2, \ldots, n$ are associated with twin systems. Denote

$\eta_{0}=1 \quad \forall \mathbf{X} \in$ parent, $\quad \eta_{i}=1 \quad \forall \mathbf{X} \in$ twin system $i$,

$0<\eta_{i}<1 \quad \forall \mathbf{X} \in$ interfaces.

The following constraint applies [3]:

$\sum_{i=0}^{n} \eta_{i}(\mathbf{X}, t)=1$.

Order parameter $\eta_{i}$ can be interpreted as the mass fraction of phase $i$ at a given material point $\mathbf{X}$. Twinning kinematics for a given system $i$ are described by the simple shear

$\hat{\mathbf{F}}_{i}=\mathbf{1}+\gamma_{0 i} \mathbf{s}_{i} \otimes \mathbf{m}_{i} \quad\left(\right.$ with $\left.\mathbf{s}_{i} \cdot \mathbf{m}_{i}=0, \mathbf{s}_{i} \cdot \mathbf{s}_{i}=\mathbf{m}_{i} \cdot \mathbf{m}_{i}=1\right)$. 
The unit normal to the surface of composition (i.e., the habit plane) in the reference configuration is $\mathbf{m}_{i}$. The magnitude of the twinning shear and the shear direction are $\gamma_{0_{i}}$ and $\mathbf{s}_{i}$, respectively. The deformation gradient $\mathbf{F}=\nabla \chi$ is decomposed as in (6):

$\mathbf{F}=\mathbf{F}^{E} \mathbf{F}^{\eta}$

where

$\mathbf{F}^{E}(\mathbf{X}, t)=\mathbf{F}\left(\mathbf{F}^{\eta}\right)^{-1} \equiv$ elastic deformation,

$\mathbf{F}^{\eta}\left[\eta_{i}(\mathbf{X}, t) ; i=1,2, \ldots, n\right] \equiv$ stress-free twinning shear.

Specifically, twinning shear is interpolated as follows:

$\mathbf{F}^{\eta}=\mathbf{1}+\sum_{i=1}^{n}\left[\varphi_{i}\left(\eta_{i}\right)\right] \gamma_{0} \mathbf{s}_{i} \otimes \mathbf{m}_{i}$,

where interpolation functions analogous to (9) are

$\varphi_{i}\left(\eta_{i}\right)=\alpha \eta_{i}^{2}+2(2-\alpha) \eta_{i}^{3}+(\alpha-3) \eta_{i}^{4}, \quad 0<\alpha<6$.

Clearly $\mathbf{F}^{\eta}\left(\eta_{i}=1 ; i>0\right)=\mathbf{1}+\gamma_{0 i} \mathbf{s}_{i} \otimes \mathbf{m}_{i}=\hat{\mathbf{F}}_{i}$ and $\mathbf{F}^{\eta}\left(\eta_{i}=0 \forall i>\right.$ $0)=\mathbf{1}$. For a single twin system, $\operatorname{det} \mathbf{F}^{\eta}=\operatorname{det}\left(\mathbf{1}+\varphi_{i} \gamma_{0} \mathbf{s}_{i} \otimes \mathbf{m}_{i}\right)=$ $1+\varphi_{i} \gamma_{0 i} \mathbf{s}_{i} \cdot \mathbf{m}_{i}=1$, so volume is conserved. However,

$$
\begin{aligned}
\frac{\mathrm{d}}{\mathrm{d} t}\left(\operatorname{det} \mathbf{F}^{\eta}\right) & =\left(\operatorname{det} \mathbf{F}^{\eta}\right) \operatorname{tr}\left[\dot{\mathbf{F}}^{\eta}\left(\mathbf{F}^{\eta}\right)^{-1}\right] \\
& \approx\left(\operatorname{det} \mathbf{F}^{\eta}\right) \sum_{i=1}^{n} \dot{\varphi} \gamma_{0_{i}} \mathbf{s}_{i} \cdot \mathbf{m}_{i}=0,
\end{aligned}
$$

so volume is only conserved approximately by twinning when multiple phases are present (e.g., at triple points). Strain energy per unit reference volume $W$ is of the functional form

$W=W\left(\mathbf{E}^{E}, \eta_{i}\right)$,

where $\mathbf{E}^{E}$ is the defined in (11). Strain energy density $W$ always vanishes at null elastic strain:

$W(\mathbf{0}, \bullet)=0$.

Strain energy density and second-order moduli are written

$W=\frac{1}{2} \mathbf{E}^{E}: \mathbb{C}\left(\eta_{i}\right): \mathbf{E}^{E}, \quad \mathbb{C}\left(\eta_{i}\right)=\left.\frac{\partial^{2} W}{\partial \mathbf{E}^{E} \partial \mathbf{E}^{E}}\right|_{\mathbf{E}^{E}=\mathbf{0}}$.

For a compound twin in a centrosymmetric lattice,

$\mathbf{Q}_{i}=2 \mathbf{m}_{i} \otimes \mathbf{m}_{i}-\mathbf{1}$,

and elastic coefficients of twins $\mathbb{C}_{i}$ are related to those of the parent $\mathbb{C}_{0}$ by

$$
\begin{aligned}
C_{i A B C D} & =C\left(\eta_{i}=1\right)_{A B C D} \\
& =Q_{i A E} Q_{i B F} Q_{i C G} Q_{i D H} C_{0 E F G H} .
\end{aligned}
$$

Elastic coefficients in the interface are interpolated the same way as the twinning shear:

$\mathbb{C}\left(\eta_{i}\right)=\mathbb{C}_{0}+\sum_{i=1}^{n}\left[\mathbb{C}_{i}-\mathbb{C}_{0}\right] \varphi_{i}$.

In the isotropic approximation, $\mathbb{C}_{0}=\mathbb{C}_{i} \forall i$ and $W$ does not depend explicitly on $\eta_{i}$. The local interfacial energy per unit reference volume is written as follows, extending (18):

$$
\begin{aligned}
f\left(\eta_{i}, \nabla \eta_{i}\right)= & \sum_{i=1}^{n}\left[f_{0 i}\left(\eta_{i}\right)+\kappa_{i}:\left(\nabla \eta_{i} \otimes \nabla \eta_{i}\right)\right] \\
& +\sum_{i=1}^{n-1} \sum_{j=i+1}^{n} g_{i j}\left(\eta_{i}, \eta_{j}\right),
\end{aligned}
$$

with $\kappa_{i}$ symmetric second-order tensors of material constants. A possibility for $f_{0 i}$ is

$f_{0 i}=A \eta_{i}^{2}\left(1-\eta_{i}\right)^{2}$ with $A$ a non-negative constant. The following function penalizes more than two phases at a boundary (e.g., at triple points) [20]:

$g_{i j}\left(\eta_{i}, \eta_{j}\right)=B \eta_{i}^{2} \eta_{j}^{2}\left(1-\eta_{i}-\eta_{j}\right)$,

with $B$ a non-negative constant. Energy function (B.15) degenerates to (18) when only one twin system is present. Other possible functions that might be considered are given by Steinbach et al. [3]. The total free energy functional $\Psi$ is defined as the volume integral

$\Psi=\int_{\Omega} W\left(\mathbf{E}^{E}, \eta_{i}\right) \mathrm{d} \Omega+\int_{\Omega} f\left(\eta_{i}, \nabla \eta_{i}\right) \mathrm{d} \Omega$.

Applying Hamilton's principle, the following variational equation is posited to suggest static equilibrium and boundary conditions:

$\delta \Psi-\int_{\partial \Omega} \mathbf{t} \cdot \delta \chi \mathrm{d} S-\sum_{i=1}^{n} \int_{\partial \Omega} h_{i} \delta \eta_{i} \mathrm{~d} S=0$,

where $h_{i}$ is a scalar conjugate force to variation in order parameter $\eta_{i}$. Taking the first variation of the interfacial energy and applying the divergence theorem, the analog of (29) is

$$
\begin{aligned}
& \delta \int_{\Omega} f \mathrm{~d} \Omega=\sum_{i=1}^{n}\left\{\int_{\Omega} \frac{\partial f_{0}}{\partial \eta_{i}} \delta \eta_{i} \mathrm{~d} \Omega\right. \\
& \left.-\int_{\Omega} 2 \boldsymbol{\kappa}_{i}:\left[\nabla\left(\nabla \eta_{i}\right)\right] \delta \eta_{i} \mathrm{~d} \Omega+\int_{\partial \Omega} 2 \boldsymbol{\kappa}_{i}:\left[\left(\nabla \eta_{i}\right) \otimes \mathbf{n}\right] \delta \eta_{i} \mathrm{~d} S\right\} \\
& \quad+\sum_{i=1}^{n-1} \sum_{j=i+1}^{n} \int_{\partial \Omega} \frac{\partial g_{i j}}{\partial \eta_{i}} \delta \eta_{i} \mathrm{~d} \Omega .
\end{aligned}
$$

Taking the variation of strain energy with $\mathbf{F}$ and $\eta_{i}$ the independent variables,

$$
\begin{aligned}
\delta \int_{\Omega} W \mathrm{~d} \Omega= & \sum_{i=1}^{n} \int_{\Omega} \frac{\partial W}{\partial \eta_{i}} \delta \eta_{i} \mathrm{~d} \Omega-\int_{\Omega}\left[\nabla \cdot \frac{\partial W}{\partial \mathbf{F}}\right] \cdot \delta \chi \mathrm{d} \Omega \\
& +\int_{\partial \Omega}\left[\mathbf{n} \cdot \frac{\partial W}{\partial \mathbf{F}}\right] \cdot \delta \chi \mathrm{d} S .
\end{aligned}
$$

For admissible variations $\delta \chi$ and $\delta \eta_{i}$, it follows that in $\Omega$, Euler-Lagrange equations are

$\left.\nabla \cdot \frac{\partial W}{\partial \mathbf{F}}\right|_{\eta_{i}}=\nabla \cdot \mathbf{P}=0$

$\frac{\partial f_{0 i}}{\partial \eta_{i}}-2 \kappa_{i}:\left[\nabla\left(\nabla \eta_{i}\right)\right]+\left.\frac{\partial W}{\partial \eta_{i}}\right|_{\mathbf{F}}+\sum_{j=i+1}^{n} \frac{\partial g_{i j}}{\partial \eta_{i}}=0$

$(i=1,2, \ldots, n)$.

Boundary conditions are

$\mathbf{t}=\mathbf{P n} ; \quad h_{i}=2 \kappa_{i}:\left(\nabla \eta_{i} \otimes \mathbf{n}\right) \quad(i=1,2, \ldots, n)$.

\section{Appendix C. Interfacial energy}

Consider the theory of Sections 2 and 3 wherein a single order parameter is sufficient. Twinned region and parent are separated by a thin interface in which $0<\eta<1$. The contribution of the phase field to the free energy density, in the absence of elastic strain energy, is written as the Taylor series expansion [1]

$$
\begin{aligned}
f(\eta, \nabla \eta, \nabla \nabla \eta, \ldots)= & f_{0}(\eta)+\left.\frac{\partial f}{\partial \nabla \eta}\right|_{0} \cdot \nabla \eta+\left.\frac{\partial f}{\partial \nabla \nabla \eta}\right|_{0}: \nabla \nabla \eta \\
& +\left.\frac{1}{2} \frac{\partial^{2} f}{\partial \nabla \eta \partial \nabla \eta}\right|_{0}:(\nabla \eta \otimes \nabla \eta)+\ldots \\
= & f_{0}(\eta)+\boldsymbol{\Lambda} \cdot \nabla \eta+\xi:(\nabla \nabla \eta) \\
& +\frac{1}{2} \zeta:(\nabla \eta \otimes \nabla \eta)+\ldots,
\end{aligned}
$$


where zero subscripts correspond to a uniform value of the order parameter. Here it is assumed for convenience that minima at uniform composition occur at wells $f_{0}(0)=f_{0}(1)=0$, and that $f_{0} \geq 0$; in terminology of Allen and Cahn [2], $\Delta f_{0}=f_{0}(\bullet)-f_{0}(0)=$ $f_{0}(\bullet)-f_{0}(1)=f_{0}(\bullet)$. The analysis is limited to centrosymmetric structures for which

$\boldsymbol{\Lambda}=\mathbf{0}$.

Other coefficients can depend on the order parameter [1]. For isotropic or cubic crystals, $\boldsymbol{\xi}=\xi \mathbf{1}$ and $\zeta=\zeta \mathbf{1}$. Integrating (C.1) over $\Omega$ and omitting higher-order terms,

$$
\begin{aligned}
\Psi & =\int_{\Omega} f \mathrm{~d} \Omega \\
& =\int_{\Omega}\left[f_{0}+\xi:(\nabla \nabla \eta)+\frac{1}{2} \zeta:(\nabla \eta \otimes \nabla \eta)\right] \mathrm{d} \Omega .
\end{aligned}
$$

Applying the divergence theorem and the chain rule,

$$
\begin{aligned}
\int_{\Omega} \xi:(\nabla \nabla \eta) \mathrm{d} \Omega= & \int_{\partial \Omega} \boldsymbol{\xi}:(\nabla \eta \otimes \mathbf{n}) \mathrm{d} S \\
& -\int_{\Omega}(\nabla \cdot \boldsymbol{\xi}) \cdot(\nabla \eta) \mathrm{d} \Omega \\
= & -\int_{\Omega} \frac{\partial \boldsymbol{\xi}}{\partial \eta}:(\nabla \eta \otimes \nabla \eta) \mathrm{d} \Omega,
\end{aligned}
$$

assuming $\nabla \eta=0$ along $\partial \Omega$ [1]. Substituting (C.4) into (C.3),

$\Psi=\int_{\Omega}\left[f_{0}+\kappa:(\nabla \eta \otimes \nabla \eta)\right] \mathrm{d} \Omega$,

where

$\kappa=\left.\left(\frac{\zeta}{2}-\frac{\partial \xi}{\partial \eta}\right)\right|_{0}=\left.\frac{1}{2} \frac{\partial^{2} f}{\partial \nabla \eta \partial \nabla \eta}\right|_{0}-\left.\frac{\partial^{2} f}{\partial \eta \partial \nabla \nabla \eta}\right|_{0}$.

For cubic or isotropic symmetry, (C.5) and (C.6) reduce to [1]

$$
\begin{gathered}
\Psi=\int_{\Omega}\left[f_{0}+\kappa|\nabla \eta|^{2}\right] \mathrm{d} \Omega, \\
\kappa=\left.\left(\frac{\zeta}{2}-\frac{\partial \xi}{\partial \eta}\right)\right|_{0}=\left.\frac{1}{2} \frac{\partial^{2} f}{\partial|\nabla \eta|^{2}}\right|_{0}-\left.\frac{\partial^{2} f}{\partial \eta \partial \nabla^{2} \eta}\right|_{0} .
\end{gathered}
$$

Consider a flat interface, for which are sought the interfacial energy and equilibrium thickness under stress-free conditions [1,2]. In the present context, these are physically related, respectively, to twin boundary energy per unit area and the thickness of the region near the boundary where atomic positions deviate from those of a perfect lattice. Henceforth in Appendix C, it is assumed that $\kappa=$ $\kappa \mathbf{1}=$ constant. At equilibrium, when strain energy $W$ vanishes, (32) gives

$$
\frac{\partial f_{0}}{\partial \eta}=2 \kappa \nabla^{2} \eta
$$

Consider an infinitely long columnar volume with element $\mathrm{d} \Omega=A_{0} \mathrm{~d} X$ having constant cross-sectional area $A_{0}$ and normal Lagrangian coordinate to the interface $X$. Assume that $\eta$ varies only in the $X$ direction: $\eta=\eta(X)$. Then (C.8) becomes

$\frac{\partial f_{0}}{\partial \eta}=2 \kappa \frac{\partial^{2} \eta}{\partial X^{2}}$

Integrating both sides and applying the chain rule [2],

$$
\begin{aligned}
\int_{0}^{\eta(X)} \frac{\partial f_{0}}{\partial \eta} \mathrm{d} \eta & =\int_{0}^{\eta(X)}\left\{\partial\left[\kappa(\partial \eta / \partial X)^{2}\right] / \partial \eta\right\} \mathrm{d} \eta \\
& \Rightarrow \partial \eta / \partial X=\left(f_{0} / \kappa\right)^{1 / 2} .
\end{aligned}
$$

Interfacial energy per unit area $\Gamma$ is defined as [1]

$$
\begin{aligned}
\Gamma & =\int_{-\infty}^{+\infty} f \mathrm{~d} X=\int_{-\infty}^{+\infty}\left[f_{0}+\kappa(\partial \eta / \partial X)^{2}\right] \mathrm{d} X \\
& =2 \int_{-\infty}^{+\infty} f_{0} \mathrm{~d} X=2 \int_{0}^{1}\left(\kappa f_{0}\right)^{1 / 2} \mathrm{~d} \eta,
\end{aligned}
$$

where limits $\eta(X \rightarrow-\infty)=0$ and $\eta(X \rightarrow+\infty)=1$. A central difference approximation of the equilibrium thickness $l$ of the interfacial region is [1]

$$
\begin{aligned}
\left.\frac{\partial \eta}{\partial X}\right|_{f_{0, \max }} & \approx\left[\eta\left(X^{+}\right)-\eta\left(X^{-}\right)\right] /\left[X^{+}-X^{-}\right] \approx 1 / l \\
& \approx\left(f_{0, \max } / \kappa\right)^{1 / 2},
\end{aligned}
$$

where for a symmetric potential $f_{0}$ with maximum at the midpoint of the interface,

$f_{0, \max }=\left.f_{0}\right|_{X^{-}+l / 2}=\left.f_{0}\right|_{X^{+}-l / 2}=\left.f_{0}\right|_{\eta=1 / 2}$.

Applying this argument to the double-well function $f_{0}$ of (20) gives the "height" of the well:

$f_{0, \max }=f_{0}(1 / 2)=A / 16$.

From (C.12), the equilibrium thickness of the interface is

$l \approx\left(\kappa / f_{0, \max }\right)^{1 / 2}=4(\kappa / A)^{1 / 2}$.

From (C.11), the equilibrium surface energy of the interface is

$$
\begin{aligned}
\Gamma & =2 \int_{0}^{1}\left(\kappa f_{0}\right)^{1 / 2} \mathrm{~d} \eta=2(A \kappa)^{1 / 2} \int_{0}^{1} \eta(1-\eta) \mathrm{d} \eta \\
& =(A \kappa)^{1 / 2} / 3 .
\end{aligned}
$$

Use of (C.15) and (C.16) results in $\kappa=3 \Gamma l / 4$ and $A=12 \Gamma / l$ as listed in (21).

\section{Appendix D. Kinetic approach}

A typical approach for modeling time evolution of the order parameter towards an equilibrium point involves replacement of static equilibrium condition (32) with a kinetic equation, following the TDGL formalism. Phase equilibrium can be expressed as

$\delta \psi / \delta \eta=\partial f_{0} / \partial \eta-2 \kappa:[\nabla(\nabla \eta)]+\left.(\partial W / \partial \eta)\right|_{\mathbf{F}}=0$.

The kinetic approach [2] suggests an evolution law of the form

$\left.(\partial \eta / \partial t)\right|_{\mathbf{x}}=\dot{\eta}=-L\{\delta \psi / \delta \eta\} ; L=$ constant $>0$.

Substituting gives

$\dot{\eta}=-L\left\{\partial f_{0} / \partial \eta-2 \kappa:[\nabla(\nabla \eta)]+\left.(\partial W / \partial \eta)\right|_{\mathbf{F}}\right\}$.

This evolution equation is supplemented by initial conditions $\eta(\mathbf{X}, 0)$ throughout domain $\Omega$. Equilibrium is approached as $\dot{\eta} \rightarrow$ 0 [2]. A similar approach can be applied in the context of multiple order parameters (Appendix B, $[3,20]$ ). In that case, from (B.23), evolution laws of the following form are suggested, where $L_{k i}=L_{i k}$ is a positive definite matrix of kinetic coefficients:

$$
\begin{aligned}
\dot{\eta}_{k}= & -L_{k i} \sum_{i=1}^{n}\left\{\frac{\partial f_{0 i}}{\partial \eta_{i}}-2 \kappa_{i}:\left[\nabla\left(\nabla \eta_{i}\right)\right]\right. \\
& \left.+\left.\frac{\partial W}{\partial \eta_{i}}\right|_{\mathbf{F}}+\sum_{j=i+1}^{n} \frac{\partial g_{i j}}{\partial \eta_{i}}\right\} .
\end{aligned}
$$

\section{Appendix E. Analytical solution, homogeneous twin nucleation}

Following Lee and Yoo [30], considered in what follows are conditions for nucleation of a two-dimensional (i.e., cylindrical) twin embedded in an otherwise homogeneous elastic solid of 
infinite extent. The analysis of Lee and Yoo [30] builds on earlier work of Eshelby [42] and Johnson and Cahn [49]. Similar analyses of the Gibbs free energy change associated with twin nucleation have since appeared [11,31-33]. The present analysis is limited to isotropic linear elasticity, with identical elastic constants shared by the twin (i.e., the inclusion) and the parent (i.e., the matrix). Consider an ellipsoidal twin with semi-axes $a_{1}, a_{2}$, and $a_{3}$, where $a_{3} \rightarrow \infty$ such that the ellipsoid degenerates to an infinitely extended cylinder. The cross-sectional area of the twin is $A_{T}=$ $\pi a_{1} a_{2}$, and the aspect ratio of the twin is $\omega=a_{2} / a_{1} \leq 1$. The total Gibbs free energy change per unit length of the cylinder associated with twin nucleation is

$\Delta G=W_{E}+\Phi_{S}-A_{T} \tau_{\infty} \gamma_{0}$.

The elastic strain energy per unit length is [41]

$W_{E}=A_{T} S \omega /(1+\omega)^{2}, \quad S=\mu \gamma_{0}^{2} /(2-2 v)$.

The surface energy per unit length of the twin embryo is [30]

$\Phi_{S}=4 \Gamma\left[A_{T} /(\pi \omega)\right] E(k, \pi / 2)$,

where the complete elliptic integral of the second kind with $k=$ $\left(1-\omega^{2}\right)^{1 / 2}$ is

$$
\begin{aligned}
E(k, \pi / 2)= & \int_{0}^{\pi / 2}\left(1-k^{2} \sin ^{2} \phi\right)^{1 / 2} \mathrm{~d} \phi \\
= & \frac{\pi}{2}\left[1-\left(\frac{1}{2}\right)^{2} k^{2}-\left(\frac{1 \cdot 3}{2 \cdot 4}\right)^{2} \frac{k^{4}}{3}\right. \\
& \left.-\left(\frac{1 \cdot 3 \cdot 5}{2 \cdot 4 \cdot 6}\right)^{2} \frac{k^{6}}{5}-\cdots\right] .
\end{aligned}
$$

The work per unit length associated with far-field loading stress $\sigma_{\infty}$ is $[30,31]$

$-A_{T} \tau_{\infty} \gamma_{0}=-A_{T}\left[\sigma_{\infty}:(\mathbf{s} \otimes \mathbf{m})\right] \gamma_{0}$.

The critical aspect ratio and critical size for twin nucleation for a given far-field stress $\sigma_{\infty}$ are determined by the simultaneous stationary conditions

$\frac{\partial \Delta G}{\partial \omega}=0, \quad \frac{\partial \Delta G}{\partial A_{T}}=0$.

Using (E.1)-(E.5), these two conditions can be expressed as [30]

$$
\begin{aligned}
\tau_{\infty} \gamma_{0} / S= & \omega /(1+\omega)^{2}+\omega(\omega-1) E \\
& \times\left\{(1+\omega)^{3}[2 \omega(\partial E / \partial \omega)-E]\right\}^{-1} .
\end{aligned}
$$

$A_{T}=\left\{2 \Gamma(1+\omega)^{3}[2 \omega(\partial E / \partial \omega)-E]\right.$

$$
\left.\times\left[\pi^{1 / 2} S \omega^{3 / 2}(\omega-1)\right]^{-1}\right\}^{2} .
$$

Simultaneous solution of (E.7) and (E.8) gives the critical aspect ratio $\omega$ and size, i.e., area $A_{T}$ or major semi-axis $a_{1}=\left[A_{T} /(\pi \omega)\right]^{1 / 2}$, of a twin nucleus for a given far-field stress $\tau_{\infty}$. Noting the solution is a saddle point [31,32] described by $\partial^{2}(\Delta G) / \partial \omega^{2}>0$ and $\partial^{2}(\Delta G) / \partial A_{T}^{2}<0$, it follows that the solution is unstable with respect to changes in size of the inclusion. For a fixed aspect ratio and fixed far-field stress, if an embryo smaller than the critical size given by (E.8) is introduced in the matrix, it will tend to disappear, while if it is larger than the critical size, it will tend to grow. The greater the applied stress $\tau_{\infty}$, the smaller the critical size, meaning that given a statistical distribution of potential twin embryo sizes and shapes, nucleation becomes more feasible as the applied stress increases [31,32]. When $\tau_{\infty}$ exceeds the following threshold, the solution is a circular cylinder [30]:

$\tau_{\infty} \geq 5 S /\left(12 \gamma_{0}\right)=5 \mu \gamma_{0} /[24(1-v)]: \omega=1$,

$A_{T}=\pi \Gamma^{2}\left[\tau_{\infty} \gamma_{0}-S / 4\right]^{-1}$.

\section{References}

[1] J. Cahn, J. Hilliard, Free energy of a nonuniform system. I. Interfacial free energy, J. Chem. Phys. 28 (1958) 258-267.
[2] S. Allen, J. Cahn, A microscopic theory for antiphase boundary motion and its application to antiphase domain coarsening, Acta Metall. 27 (1979) 1085-1095.

[3] I. Steinbach, F. Pezzolla, B. Nestler, M. Seebelberg, R. Prieler, G. Schmitz, J. Rezende, A phase field concept for multiphase systems, Physica D 94 (1996) 135-147.

[4] I. Steinbach, M. Apel, Multi phase field model for solid state transformation with elastic strain, Physica D 217 (2006) 153-160.

[5] E. Fried, M. Gurtin, Dynamic solid-solid transitions with phase characterized by an order parameter, Physica D 72 (1994) 287-308.

[6] M. Gurtin, Generalized Ginzburg-Landau and Cahn-Hilliard equations based on a microforce balance, Physica D 92 (1996) 178-192.

[7] N. Moelans, B. Blanpain, P. Wollants, An introduction to phase-field modeling of microstructure evolution, Calphad 32 (2008) 268-294.

[8] I. Singer-Loginova, H. Singer, The phase field technique for modeling multiphase materials, Rep. Prog. Phys. 71 (2008) 106501.

[9] A. Kosevich, V. Boiko, Dislocation theory of the elastic twinning of crystals, Sov. Phys. Uspekhi 14 (1971) 286-316.

[10] R. James, Finite deformation by mechanical twinning, Arch. Ration. Mech. Anal. 77 (1981) 143-176.

[11] J. Christian, S. Mahajan, Deformation twinning, Prog. Mater. Sci. 39 (1995) $1-157$.

[12] J. Hirth, J. Lothe, Theory of Dislocations, second edition, John Wiley and Sons, New York, 1982.

[13] J. Wang, J. Hirth, C. Tomé, (1012) twinning nucleation mechanisms in hexagonal-close-packed crystals, Acta Mater. 57 (2009) 5521-5530.

[14] J. Wang, R. Hoagland, J. Hirth, L. Capolungo, I. Beyerlein, C. Tomé, Nucleation of a (1012) twin in hexagonal close-packed crystals, Scripta Mater. 61 (2009) 903-906.

[15] C. Tomé, H.-R. Wenk, G. Canova, U. Kocks, Simulations of texture development in calcite: comparison of polycrystal plasticity theories, J. Geophys. Res. 96 (1991) 11865-11875.

[16] J. Clayton, A continuum description of nonlinear elasticity, slip, and twinning, with application to sapphire, Proc. R. Soc. Lond. A 465 (2009) 307-334.

[17] P. Rosakis, H. Tsai, Dynamic twinning processes in crystals, Internat. J. Solids Structures 32 (1995) 2711-2723.

[18] T. Hou, P. Rosakis, P. LeFloch, A level-set approach to the computation of twinning and phase-transition dynamics, J. Comput. Phys. 150 (1999) 302-331.

[19] D. Fan, L.-Q. Chen, Computer simulation of twin formation during the displacive $c \rightarrow t^{\prime}$ phase transformation in the zirconia-yttria system, J. Amer. Ceram. Soc. 78 (1995) 769-773.

[20] V. Levitas, V. Levin, K. Zingerman, E. Freiman, Displacive phase transitions at large strains: phase-field theory and simulations, Phys. Rev. Lett. 103 (2009) 025702 .

[21] J. Knap, K. Sieradzki, Crack tip dislocation nucleation in fcc solids, Phys. Rev. Lett. 82 (1999) 1700-1703.

[22] J. Knap, M. Ortiz, Effect of indenter-radius size on au(001) nanoindentation, Phys. Rev. Lett. 90 (2003) 226102.

[23] A. Serra, R. Pond, D. Bacon, Computer simulation of the structure and mobility of twinning dislocations in h.c.p. metals, Acta Metall. Mater. 39 (1991) 1469-1480.

[24] A. Serra, D. Bacon, Computer simulation of twinning dislocation in magnesium using a many-body potential, Phil. Mag. A 63 (1991) 1001-1012.

[25] A. Paxton, P. Gumbsch, M. Methfessel, A quantum mechanical calculation of the theoretical strength of metals, Phil. Mag. Lett. 63 (1991) 267-274.

[26] A. Idesman, V. Levitas, E. Stein, Elastoplastic materials with martensitic phase transition and twinning at finite strains: numerical solution with finite element method, Comput. Methods Appl. Mech. Engrg. 173 (1999) 71-98.

[27] M. Koslowski, A. Cuitino, M. Ortiz, A phase-field theory of dislocation dynamics, strain hardening, and hysteresis in ductile single crystals, J. Mech. Phys. Solids 50 (2002) 2597-2635.

[28] P. Gumbsch, H. Gao, Dislocations faster than the speed of sound, Science 283 (1999) 965-968.

[29] R. Cooper, Kinetics of elastic twinning in calcite, Proc. R. Soc. Lond. A 270 (1962) 525-537.

[30] J. Lee, M. Yoo, Elastic strain energy of deformation twinning in tetragonal crystals, Metall. Trans. A 21 (1990) 2521-2530.

[31] M. Yoo, J. Lee, Deformation twinning in h.c.p. metals and alloys, Phil. Mag. 63 (1991) 987-1000.

[32] R. Lebensohn, C. Tomé, A study of the stress state associated with twin nucleation and propagation in anisotropic materials, Phil. Mag. A 67 (1993) 187-206.

[33] M. Meyers, O. Vohringer, V. Lubarda, The onset of twinning in metals: a constitutive description, Acta Mater. 49 (2001) 4025-4039.

[34] A. Staroselsky, L. Anand, A constitutive model for hcp materials deforming by slip and twinning: application to magnesium alloy az31b, Int. J. Plasticity 19 (2003) 1843-1864

[35] V. Levitas, D. Preston, Three-dimensional landau theory for multivariant stress-induced martensitic phase transformations. I. Austenite $\leftrightarrow$ martensite, Phys. Rev. B 66 (2002) 134206.

[36] J. Clayton, D. Bammann, D. McDowell, A geometric framework for the kinematics of crystals with defects, Phil. Mag. 85 (2005) 3983-4010.

[37] R. Thurston, Waves in solids, in: C. Truesdell (Ed.), Handbuch der Physik Via/4, Springer-Verlag, Berlin, 1974, pp. 109-308. 
[38] I. Gelfand, S. Fomin, Calculus of Variations, Dover, Mineola, New York, 2000

[39] B. Dacorogna, Direct Methods in the Calculus of Variations, Springer, New York, 1989.

[40] P. Ciarlet, Mathematical Elasticity, North-Holland, Amsterdam, 1988.

[41] J. Eshelby, The determination of the elastic field of an ellipsoidal inclusion, and related problems, Proc. R. Soc. Lond. A 241 (1957) 376-396.

[42] J. Eshelby, Elastic inclusions and inhomogeneities, in: I. Sneddon, R. Hill (Eds.), in: Progress in Solid Mechanics, vol. 2, North-Holland, Amsterdam, 1961, pp. 89-140.

[43] E. Orowan, Dislocations and mechanical properties, in: M. Cohen (Ed.), Dislocations in Metals, American Institute of Mining and Metallurgical Engineers, New York, 1954, pp. 69-195.
[44] L. Slutsky, C. Garland, Elastic constants of magnesium from 4.2 to $300 \mathrm{~K}$, Phys Rev. 107 (1957) 972-976.

[45] M. Kronberg, A structural mechanism for the twinning process on $\{1012\}$ in hexagonal close packed metals, Acta. Metall. 16 (1968) 29-34.

[46] R. Hill, The elastic behavior of a crystalline aggregate, Proc. Phys. Soc. Lond. A 65 (1952) 349-354.

[47] R. Hearmon, The elastic constants of anisotropic materials, Rev. Modern Phys 18 (1946) 409-439.

[48] R. Bell, R. Cahn, The dynamics of twinning and the interrelation of slip and twinning in zinc crystals, Proc. R. Soc. Lond. A 239 (1957) 494-521.

[49] W. Johnson, J. Cahn, Elastically induced shape bifurcations of inclusions, Acta Metall. 32 (1984) 1925-1933. 